\title{
SOLVING THE CAHN-HILLIARD VARIATIONAL INEQUALITY WITH A SEMI-SMOOTH NEWTON METHOD
}

\author{
Luise BlanK ${ }^{1}$, Martin Butz ${ }^{1}$ And Harald Garcke ${ }^{1}$
}

\begin{abstract}
The Cahn-Hilliard variational inequality is a non-standard parabolic variational inequality of fourth order for which straightforward numerical approaches cannot be applied. We propose a primal-dual active set method which can be interpreted as a semi-smooth Newton method as solution technique for the discretized Cahn-Hilliard variational inequality. A (semi-)implicit Euler discretization is used in time and a piecewise linear finite element discretization of splitting type is used in space leading to a discrete variational inequality of saddle point type in each time step. In each iteration of the primal-dual active set method a linearized system resulting from the discretization of two coupled elliptic equations which are defined on different sets has to be solved. We show local convergence of the primal-dual active set method and demonstrate its efficiency with several numerical simulations.
\end{abstract}

Mathematics Subject Classification. 35K55, 35K85, 90C33, 49N90, 80A22, 82C26, 65M60.

Received June 18, 2009. Revised March 2, 2010.

Published online August 18, 2010.

\section{INTRODUCTION}

The Cahn-Hilliard equation was initially introduced to model phase separation in binary alloys [10]. By now the Cahn-Hilliard equation has found many applications ranging from classical aspects in materials science [25,38] over image processing [11], fluid dynamics [37], topology optimization [44] up to the modelling of mineral growth [34] and galaxy structure formation [42]. The Cahn-Hilliard equation can model interface motion in so called conserved systems, i.e. in systems where the concentration of a species or the volume occupied by a phase is conserved. In these applications Cahn-Hilliard variational inequalities are frequently used.

Commonly the Cahn-Hilliard variational inequality is solved numerically by (semi-)implicit in time discretizations leading to a variational inequality of saddle point type. Iterative solution techniques for the resulting system are simple recursive methods using a semi-smooth reformulation of the complementary formulation of the variational inequality, see [7], splitting methods using an approach of Lions and Mercier $[3,14,35]$ and nonlinear Gauss-Seidel and SOR methods $[4,20]$. Only recently multigrid methods for the discrete variational inequality have been investigated, see [2,27-29] and the discussion below.

In this paper we propose a numerical method for solving Cahn-Hilliard variational inequalities and we heavily use the gradient flow structure of the Cahn-Hilliard model. We interpret the time discretized version of the gradient flow as a PDE-constraint optimization problem where in addition pointwise inequality constraints have

Keywords and phrases. Cahn-Hilliard equation, active-set methods, semi-smooth Newton methods, gradient flows, PDE-constraint optimization, saddle point structure.

${ }^{1}$ Universität Regensburg, NWF I-Mathematik, 93040 Regensburg, Germany. luise.blank@mathematik.uni-regensburg.de 
to hold. The PDE-constraint minimization problem which we obtain is non-standard as the objective functional contains the $L^{2}$-norms of gradients rather than the $L^{2}$-norms of the involved functions itself. We propose to solve the fully discretized system with a primal-dual active set method which can be reinterpreted as a semi-smooth Newton method. It turns out that this approach is superior to earlier numerical methods for Cahn-Hilliard variational inequalities. Its efficiency in each iteration step is comparable to a method proposed by Gräser and Kornhuber [26-28]. They introduce and analyse an Uzawa-multigrid algorithm for set-valued saddle point problems for which also global convergence results are shown [28]. There in each iteration step an intermediate primal-active set is determined with the help of obstacle problems which are solved with a monotone multigrid method. In a second step a linear subproblem similar to ours where only the right hand side differs has to be solved and then the chemical potential is updated by a damped gradient-type method. After convergence the phase field is determined. In contrast we determine the active sets in a simple way using approximations of the primal and dual variables. Then we solve the same linear subproblem as in [26-28] where we compute for the chemical potential and the phase field simultaneously. Banas and Nürnberg [2] applied a fully nonlinear multigrid method to the whole system of Cahn-Hilliard inequalities and exhibit mesh-independent convergence properties. Computational comparisons are not available up to now.

The outline of the paper is as follows. In the remainder of this section we introduce the Cahn-Hilliard variational inequality. We will interpret the implicit time discretization of the Cahn-Hilliard variational inequality as a PDE-constraint optimization problem in Section 2. In Section 3 we introduce a primal-dual active set approach for the time discretized Cahn-Hilliard variational inequality and we formulate a finite element method for a splitting formulation of the Cahn-Hilliard variational inequality in Section 4. We also show local convergence. Finally, we numerically analyse the behaviour of the method with the help of four examples of different type and show some simulations in Section 5.

Since the gradient flow perspective is important for what follows we choose to derive the Cahn-Hilliard equations as a gradient flow. We remark that our derivation will be formal. We consider a vector space $\mathbf{Z}$ and an affine subspace $\mathbf{U} \subset \mathbf{Z}$, i.e. there exists a $\bar{u} \in \mathbf{Z}$ and a linear space $\mathbf{Y} \subset \mathbf{Z}$ such that $\mathbf{U}=\bar{u}+\mathbf{Y}$. The gradient of a sufficiently smooth function $E: \mathbf{U} \rightarrow \mathbb{R}$ depends on the inner product chosen for $\mathbf{Z}$. We define the first variation of $E$ at a point $u \in \mathbf{U}$ in a direction $v \in \mathbf{Y}$ by

$$
\frac{\delta E}{\delta u}(u)(v):=\lim _{\delta \rightarrow 0} \frac{E(u+\delta v)-E(u)}{\delta} .
$$

We say that there exists a gradient of $E$ with respect to the inner product $(.,.) \mathbf{z}$ on $\mathbf{Z}$ which we denote by $\operatorname{grad}_{\mathbf{z}} E(u)$ if

$$
\left.\operatorname{(grad}_{\mathbf{Z}} E(u), v\right)_{\mathbf{z}}=\frac{\delta E}{\delta u}(u)(v) \quad \text { for all } v \in \mathbf{Y} .
$$

Now the gradient flow of $E$ with respect to the inner product $(.,.) \mathbf{z}$ is given as

$$
\partial_{t} u(t)=-\operatorname{grad}_{\mathbf{Z}} E(u(t)) .
$$

The energy decreases in time due to the inequality

$$
\frac{\mathrm{d}}{\mathrm{d} t} E(u(t))=\left(\operatorname{grad}_{\mathbf{Z}} E(u(t)), \partial_{t} u(t)\right)_{\mathbf{z}}=-\left\|\partial_{t} u\right\|_{\mathbf{Z}}^{2} \leq 0 .
$$

In the following, in order to derive the Cahn-Hilliard equation, we introduce the Ginzburg-Landau energy $E: H^{1}(\Omega) \rightarrow \mathbb{R}$ as

$$
E(u)=\int_{\Omega}\left\{\frac{\gamma \varepsilon}{2}|\nabla u|^{2}+\frac{1}{\varepsilon} \psi(u)\right\} \mathrm{d} x
$$




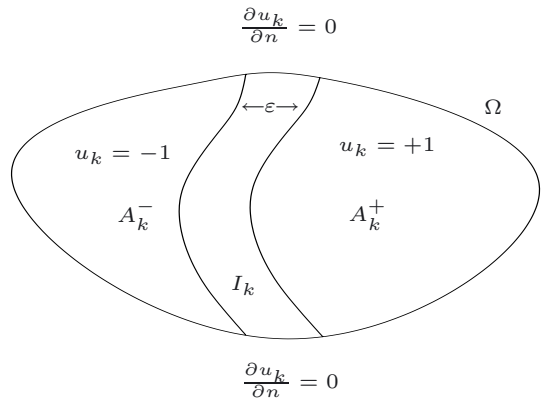

FigurE 1. The system (4.8)-(4.9) leads to an equation for $u_{k}$ on the inactive set $\mathcal{I}_{k}$ and for $w_{k}$ on the whole of $\Omega$.

where $\Omega \subset \mathbb{R}^{d}$ is a bounded domain with Lipschitz boundary, $\gamma>0$ is a constant related to the interfacial energy density and $\psi$ is a double well potential, e.g. $\psi(u)=\left(1-u^{2}\right)^{2}$ or an obstacle potential, e.g.

$$
\psi(u)=\left\{\begin{array}{ll}
\psi_{0}(u) & u \in[-1,1] \\
\infty & \text { elsewhere }
\end{array}\right\}=\psi_{0}(u)+I_{[-1,1]}(u)
$$

where $\psi_{0}$ is smooth and $I_{[-1,1]}$ is the indicator function, i.e. $I_{[-1,1]}$ is set to infinity outside the interval [-1,1] and to 0 on $[-1,1]$. For the Cahn-Hilliard model this double obstacle formulation was introduced by Blowey and Elliott [6] and we will hence refer to it as the Cahn-Hilliard model with Blowey-Elliott potential. In the following we will choose

$$
\psi_{0}(u)=\frac{1}{2}\left(1-u^{2}\right)
$$

which is the typical choice in the literature, see e.g. $[6,9,21]$, but other non-convex functions are possible. In the Cahn-Hilliard model the different phases correspond to the values $u= \pm 1$. On an interface a solution rapidly changes from values close to 1 to values close to -1 and the thickness of this interfacial region is proportional to the parameter $\varepsilon$ in (1.2), see e.g. Figure 1 .

If $\psi$ is smooth the first variation of $E$ in a direction $v$ is given as

$$
\frac{\delta E}{\delta u}(u)(v)=\int_{\Omega}\left(\gamma \varepsilon \nabla u \cdot \nabla v+\frac{1}{\varepsilon} \psi^{\prime}(u) v\right)
$$

Choosing $\mathbf{Z}=L^{2}(\Omega), \mathbf{U}=\mathbf{Y}=H^{1}(\Omega)$ and $\bar{u}=0$ we obtain

$$
\operatorname{grad}_{L^{2}} E(u)=-\gamma \varepsilon \Delta u+\frac{1}{\varepsilon} \psi^{\prime}(u)
$$

and the resulting gradient flow equation gives the so called Allen-Cahn equation. We remark here that for (1.6) to hold we also need to require $\frac{\partial u}{\partial n}=0$ on $\partial \Omega$ where $n$ is the outer unit normal to $\partial \Omega$.

As mentioned above in the Cahn-Hilliard model the total concentration, i.e. $\int_{\Omega} u(x) \mathrm{d} x$ is assumed to be conserved. Denoting by $\underset{\Omega}{f} u$ the mean value of a function $u$, we now define for a given $m \in(-1,1)$ the sets

$$
\mathbf{U}:=\left\{u \in H^{1}(\Omega) \mid \underset{\Omega}{f} u=m\right\}, \quad \mathbf{Y}:=\left\{u \in H^{1}(\Omega) \mid \underset{\Omega}{f_{\Omega}} u=0\right\} .
$$

In addition we introduce $\mathbf{Z}=H^{-1}(\Omega)=\left\{u^{\prime} \in\left(H^{1}(\Omega)\right)^{\prime} \mid\left\langle u^{\prime}, 1\right\rangle=0\right\}$, i.e. all bounded linear functionals on $H^{1}(\Omega)$ that vanish on constant functions. Here and in what follows $\langle.,$.$\rangle denotes the dual pairing. On$ $\mathbf{Z}=H^{-1}(\Omega)$ we define the $H^{-1}$-inner product for $v_{1}, v_{2} \in \mathbf{Z}$ as

$$
\left(v_{1}, v_{2}\right)_{H^{-1}}:=\int_{\Omega} \nabla(-\Delta)^{-1} v_{1} \cdot \nabla(-\Delta)^{-1} v_{2}
$$


where $y=(-\Delta)^{-1} v$ is the weak solution of $-\Delta y=v$ in $\Omega$ and $\frac{\partial y}{\partial n}=0$ on $\partial \Omega$, i.e. $\int_{\Omega} \nabla y \cdot \nabla \eta=v(\eta)$ for all $\eta \in H^{1}(\Omega)$. We remark that the solution to this elliptic problem is only defined up to a constant and we always choose $y$ such that $f_{\Omega} y=0$. The function space $\mathbf{Y}$ is canonically embedded into $\mathbf{Z}$ since $u \in \mathbf{Y}$ can be related to the linear functional $y \mapsto \int_{\Omega} u y$. For $v_{1}, v_{2} \in \mathbf{Y}$ we obtain using the $L^{2}$-inner product (.,.)

$$
\left(v_{1}, v_{2}\right)_{H^{-1}}=\left(v_{1},(-\Delta)^{-1} v_{2}\right)=\left((-\Delta)^{-1} v_{1}, v_{2}\right) .
$$

These identities also hold more generally for functions $v_{1}, v_{2} \in L^{2}(\Omega)$ with mean value zero. To compute the $H^{-1}$-gradient of $E$ we now need to find $\operatorname{grad}_{H^{-1}} E(u) \in \mathbf{Z}$ such that

$$
\left(v, \operatorname{grad}_{H^{-1}} E(u)\right)_{H^{-1}}=\frac{\delta E}{\delta u}(u)(v) \text { holds for all } v \in \mathbf{Y} .
$$

From the above we obtain $\left(v,(-\Delta)^{-1} \operatorname{grad}_{H^{-1}} E(u)\right)=\left(v, \operatorname{grad}_{L^{2}} E(u)\right)$ and hence

$$
\operatorname{grad}_{H^{-1}} E(u)=(-\Delta) \operatorname{grad}_{L^{2}} E(u) .
$$

Then, the Cahn-Hilliard equation is given as the $H^{-1}$-gradient flow of the Ginzburg-Landau energy E. If $\psi$ is smooth we obtain the fourth order parabolic equation

$$
\partial_{t} u=-\operatorname{grad}_{H^{-1}} E(u)=\Delta\left(-\gamma \varepsilon \Delta u+\frac{1}{\varepsilon} \psi^{\prime}(u)\right)
$$

or equivalently introducing the so called chemical potential $w$ the equation can be rewritten as a system as follows

$$
\begin{aligned}
\partial_{t} u & =\Delta w, \\
w & =-\gamma \varepsilon \Delta u+\frac{1}{\varepsilon} \psi^{\prime}(u) .
\end{aligned}
$$

In addition the boundary conditions $\frac{\partial u}{\partial n}=\frac{\partial w}{\partial n}=0$ on $\partial \Omega$ have to hold. Let us remark, that in this formulation we do not necessarily have $f_{\Omega} w=0$, i.e. in general $w \neq-(-\Delta)^{-1} \partial_{t} u$ but both functions only differ by an additive constant.

It is also possible to derive the Cahn-Hilliard equation from the mass balance law and in this case $-\nabla w$ is the mass flux where for simplicity a mobility coefficient was taken to be one, see e.g. Elliott [19] or Novick-Cohen [38].

The presentation so far is appropriate for smooth functions $\psi$. If the energy has the double obstacle form (1.3) we differentiate $I_{[-1,1]}$ in the sense of subdifferentials, i.e. for a $u \in L^{2}(\Omega)$ with $|u| \leq 1$ we obtain that $\mu \in L^{2}(\Omega)$ is in the subdifferential of $I_{[-1,1]}$ at $u$ if and only if

$$
\mu \in \partial I_{[-1,1]}(u)= \begin{cases}(-\infty, 0] & \text { if } u=-1, \\ 0 & \text { for } u \in(-1,1), \\ {[0, \infty)} & \text { if } u=1,\end{cases}
$$

is fulfilled pointwise almost everywhere. This can be rewritten in the following complementarity form

$$
\mu=\mu_{+}-\mu_{-}, \mu_{+} \geq 0, \mu_{-} \geq 0, \mu_{+}(u-1)=0, \mu_{-}(u+1)=0
$$

which also has to hold almost everywhere. In this case the $H^{-1}$-gradient flow has the form

$$
\begin{aligned}
\partial_{t} u & =\Delta w \\
w & =-\gamma \varepsilon \Delta u+\frac{1}{\varepsilon}\left(\psi_{0}^{\prime}(u)+\mu\right)
\end{aligned}
$$


with $\mu \in \partial I_{[-1,1]}(u),|u| \leq 1$ and zero Neumann boundary conditions for $u$ and $w$. This formulation can be restated in a variational inequality formulation, see e.g. Blowey and Elliott [6], Kinderlehrer and Stampacchia [33] and Friedman [24] for other obstacle problems, as follows:

$$
\begin{aligned}
\partial_{t} u & =\Delta w \\
(w, \xi-u) & \leq \gamma \varepsilon(\nabla u, \nabla(\xi-u))+\frac{1}{\varepsilon}\left(\psi_{0}^{\prime}(u), \xi-u\right) \quad \forall \xi \in H^{1}(\Omega),|\xi| \leq 1,
\end{aligned}
$$

together with $|u| \leq 1$ a.e. This system is the variational inequality formulation of the Cahn-Hilliard model with a Blowey-Elliott potential. It can be shown that a unique solution $(u, w)$ exists to (1.16), (1.17). More precisely the following theorem, see [6], is true.

Theorem 1.1. Assume $\Omega$ is convex or $\partial \Omega \in C^{1,1}, u_{0} \in H^{1}(\Omega)$ with $\left|u_{0}\right| \leq 1$ and $\underset{\Omega}{f} u_{0}=m \in(-1,1)$. Then there exists a unique pair $(u, w)$ such that

$$
u \in H^{1}\left(0, T ;\left(H^{1}(\Omega)\right)^{\prime}\right) \cap L^{2}\left(0, T ; H^{2}(\Omega)\right) \cap L^{\infty}\left(0, T ; H^{1}(\Omega)\right),
$$

$|u| \leq 1$ a.e. and $w \in L^{2}\left(0, T ; H^{1}(\Omega)\right)$ which solves (using the duality pairing $\langle.,$.$\rangle between H^{-1}(\Omega)$ and $H^{1}(\Omega)$ )

$$
\left\langle\partial_{t} u, \eta\right\rangle+(\nabla w, \nabla \eta)=0 \text { for all } \eta \in H^{1}(\Omega) \text { and } t \in(0, T) \text { a.e. }
$$

together with the variational inequality $(1.17)$ and $u(0, \cdot)=u_{0}$.

In particular $\mu=\varepsilon w+\gamma \varepsilon^{2} \Delta u-\psi_{0}^{\prime}(u) \in L^{2}\left(\Omega_{T}\right)$.

\section{Cahn-Hilliard variational inequalities and PDE-Constraint optimization}

Given discrete times $t_{n}=n \tau, n \in \mathbb{N}_{0}$, where $\tau>0$ is a given time step the backward Euler discretization of the gradient flow equation (1.1) is given as

$$
\frac{1}{\tau}\left(u^{n}-u^{n-1}\right)=-\operatorname{grad}_{H^{-1}} E\left(u^{n}\right) .
$$

This time discretization has a natural variational structure. In fact one can compute a solution $u^{n}$ as the solution of the minimization problem

$$
\min _{u \in \mathbf{U}}\left\{E(u)+\frac{1}{2 \tau}\left\|u-u^{n-1}\right\|_{H^{-1}}^{2}\right\}
$$

One hence tries to decrease the energy $E$ but has to take into account that deviations from the solution at the old time step costs where the cost depends on the norm on $\mathbf{Z}=H^{-1}(\Omega)$. As Euler-Lagrange equation for (2.2) we obtain the backward Euler discretization (2.1). We remark here that (2.1) might have solutions which are not necessarily global minimizers of the minimization problem (2.2). In the case of the Cahn-Hilliard model we need to minimize

$$
\int_{\Omega}\left\{\frac{\gamma \varepsilon}{2}|\nabla u|^{2}+\frac{1}{\varepsilon} \psi(u)\right\} \mathrm{d} x+\frac{1}{2 \tau}\left\|u-u^{n-1}\right\|_{H^{-1}}^{2}
$$

under all $u \in H^{1}(\Omega)$ with $\underset{\Omega}{f} u=\underset{\Omega}{f} u^{n-1}=m$. In order to compute the $H^{-1}$-norm of $u-u^{n-1}$ we need to solve a Poisson problem and hence we obtain, in the case of the obstacle potential, the following PDE-constraint 
optimization problem

$$
\begin{gathered}
\min \left\{\frac{\gamma \varepsilon}{2} \int_{\Omega}|\nabla u|^{2}+\frac{1}{\varepsilon} \int_{\Omega} \psi_{0}(u)+\frac{\tau}{2} \int_{\Omega}|\nabla v|^{2}\right\} \\
\tau \Delta v=u-u^{n-1}, \\
\text { such that } \quad \leq 1, \underset{\Omega}{f} u=m, \\
\text { with } \frac{\partial v}{\partial n}=0 \text { on } \partial \Omega \text { and } \underset{\Omega}{f} v=0 .
\end{gathered}
$$

This formulation has the form of an optimal control problem where $u$ is the control and $v$ is the state.

We now introduce Lagrange multipliers $w \in H^{1}(\Omega)$ for the weak formulation of (2.5), and $\kappa \in \mathbb{R}$ for $\underset{\Omega}{f} v=0$ and define the Lagrangian

$$
\mathcal{L}(u, v, w, \lambda):=\frac{\gamma \varepsilon}{2} \int_{\Omega}|\nabla u|^{2}+\frac{1}{\varepsilon} \int_{\Omega} \psi_{0}(u)+\frac{\tau}{2} \int_{\Omega}|\nabla v|^{2}-\int_{\Omega} \tau \nabla w \cdot \nabla v-\int_{\Omega}\left(u-u^{n-1}\right) w-\kappa \int_{\Omega} v .
$$

With the help of this Lagrangian the equality constraints are incorporated. In fact the equality constraints are obtained as the first variation of $\mathcal{L}$ with respect to $w$ and $\kappa$. In particular, we obtain $\underset{\Omega}{f_{\Omega}} u=f_{\Omega} u^{n-1}=m$ if we vary $w$ by a constant. This implies that $w$ acts as a Lagrange multiplier simultaneously for the equality

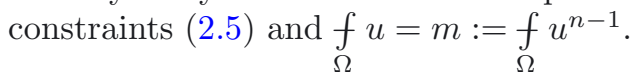

Introducing appropriately scaled Lagrange multipliers $\mu$, namely with $\frac{1}{\varepsilon}$, for the pointwise box-constraints we obtain the following version of the KKT-system where (2.8), (2.10) and (2.11) have to be understood in its weak form:

$$
\begin{aligned}
& \tau \Delta(w-v)= \kappa \text { in } \Omega, \frac{\partial w}{\partial n}=\frac{\partial v}{\partial n} \text { on } \partial \Omega, \\
& f v= 0, \kappa=0, \\
& \Omega \Delta v \text { in } \Omega, \frac{\partial v}{\partial n}=0 \text { on } \partial \Omega, \\
& \frac{1}{\tau}\left(u-u^{n-1}\right)= 0 \text { in } \Omega, \frac{\partial u}{\partial n}=0 \text { on } \partial \Omega, \\
& w+\gamma \varepsilon \Delta u-\frac{1}{\varepsilon} \psi_{0}^{\prime}(u)-\frac{1}{\varepsilon} \mu= \mu_{+}-\mu_{-}, \mu_{+} \geq 0, \quad \mu_{-} \geq 0, \text { a.e. in } \Omega, \\
& \mu 0, \mu_{-}(u+1)=0, \text { a.e. in } \Omega, \\
& \mu_{+}(u-1)=1 \text { a.e. in } \Omega .
\end{aligned}
$$

Given (2.8)-(2.9) we obtain $w-f_{\Omega} w=v$, i.e. $v$ and $w$ only differ by a constant. We can replace $v$ by $w$ in (2.10) and we hence obtain in particular a time discretization of (1.14), (1.15) using the complementary formulation (1.13). The Lagrange multiplier $w$ coincides with the chemical potential, and the scaled Lagrange multiplier $\mu$ lies in the subdifferential of $I_{[-1,1]}$. Since the equations (2.8) and (2.9) are not needed we omit them in the following.

From now on we consider the choice $\psi_{0}(u)=\frac{1}{2}\left(1-u^{2}\right)$ and show that the system $(2.10)-(2.14)$ has a solution. Defining the admissible set

$$
\mathbf{U}_{\mathbf{a d}}:=\left\{u \in H^{1}(\Omega)|| u \mid \leq 1, \underset{\Omega}{f} u=m\right\}
$$

the minimization problem (2.3) or respectively (2.4)-(2.7) can be reformulated as

$$
\min _{u \in \mathbf{U}_{\mathbf{a d}}} E(u):=\left\{\frac{\gamma \varepsilon}{2} \int_{\Omega}|\nabla u|^{2}+\frac{1}{\varepsilon} \int_{\Omega} \psi_{0}(u)+\frac{1}{2 \tau} \int_{\Omega}\left|\nabla(-\Delta)^{-1}\left(u-u^{n-1}\right)\right|^{2}\right\} .
$$


Since $\frac{1}{2}\left(1-u^{2}\right)$ is nonconvex the above minimization problem will in general omit more than one solution. But one can show that for small $\tau>0$ the problem is uniquely solvable. This is the content of the following lemma. Similar restrictions on the time step in order to obtain uniqueness had to be imposed in a fully discrete situation in $[3,7]$.

Lemma 2.1. The minimization problem (2.15) has a solution. A unique solution exists if $\tau \in\left(0,4 \gamma \varepsilon^{3}\right)$.

Proof. Since $|u| \leq 1$ we obtain that $\int_{\Omega} \psi_{0}(u)=\int_{\Omega} \frac{1}{2}\left(1-u^{2}\right)$ is non-negative and there exists a minimizing sequence $\left(u_{k}\right)_{k \in \mathbb{N}} \subset \mathbf{U}_{\text {ad }}$ for $E$, i.e.

$$
E\left(u_{k}\right) \rightarrow \inf _{u \in \mathbf{U}_{\mathrm{ad}}} E(u) \geq 0 \quad \text { for } \quad k \rightarrow \infty .
$$

Given that $\left(E\left(u_{k}\right)\right)_{k \in \mathbb{N}}$ is uniformly bounded we can conclude that $\int_{\Omega}\left|\nabla u_{k}\right|^{2} \mathrm{~d} x$ is uniformly bounded. Due to $f\left(u_{k}-m\right) \mathrm{d} x=0$ we can use Poincaré's inequality for functions with mean value zero, see e.g. [1], to obtain that $\left(u_{k}\right)_{k \in \mathbb{N}}$ is a bounded sequence in $H^{1}(\Omega)$. Using the fact that bounded sequences in $H^{1}(\Omega)$ have weakly converging subsequences and applying Rellichs theorem we obtain the existence of a subsequence such that

$$
u_{k_{j}} \rightarrow u^{*} \text { in } H^{1}(\Omega), u_{k_{j}} \rightarrow u^{*} \text { in } L^{2}(\Omega) \text { for } j \rightarrow \infty \text {. }
$$

Since the terms $\int_{\Omega}|\nabla u|^{2} \mathrm{~d} x$ and $\int_{\Omega}\left|\nabla(-\Delta)^{-1} u\right|^{2} \mathrm{~d} x$ are convex, we obtain that they are weakly lower semicontinuous in $H^{1}(\Omega)$, see e.g. [23]. Since $\int_{\Omega} \psi_{0}\left(u_{k_{j}}\right)$ converges strongly we conclude that $u^{*}$ is in fact a minimum of $E$ in $\mathbf{U}_{\mathbf{a d}}$, compare [23].

The functional $E$ is strictly convex on $\mathbf{U}$ if and only if $F(\eta):=E\left(\eta+u^{n-1}\right)$ is strictly convex on $\mathbf{Y}$. Since $F$ is the sum of terms which are constant or linear and of

$$
\frac{\gamma \varepsilon}{2} \int_{\Omega}|\nabla \eta|^{2}-\frac{1}{2 \varepsilon} \int_{\Omega} \eta^{2}+\frac{1}{2 \tau} \int_{\Omega}\left|\nabla(-\Delta)^{-1} \eta\right|^{2}
$$

we only need to show that (2.16) is strictly positive on $\mathbf{Y} \backslash\{0\}$. Using the definition of $(-\Delta)^{-1}$ and Young's inequality we obtain for all $\eta \in \mathbf{Y}$

$$
\frac{1}{2 \varepsilon} \int_{\Omega} \eta^{2}=\frac{1}{2 \varepsilon} \int_{\Omega}\left(\nabla(-\Delta)^{-1} \eta\right) \cdot \nabla \eta \leq \frac{\delta}{4 \varepsilon} \int_{\Omega}\left|\nabla(-\Delta)^{-1} \eta\right|^{2}+\frac{1}{4 \delta \varepsilon} \int_{\Omega}|\nabla \eta|^{2} .
$$

Choosing $\delta=\frac{2 \varepsilon}{\tau}$ we obtain

$$
\frac{\gamma \varepsilon}{2} \int_{\Omega}|\nabla \eta|^{2}-\frac{1}{2 \varepsilon} \int_{\Omega} \eta^{2}+\frac{1}{2 \tau} \int_{\Omega}\left|\nabla(-\Delta)^{-1} \eta\right|^{2} \geq\left(\frac{\gamma \varepsilon}{2}-\frac{\tau}{8 \varepsilon^{2}}\right) \int_{\Omega}|\nabla \eta|^{2} .
$$

If $\tau<4 \gamma \varepsilon^{3}$ we obtain uniqueness from the strict convexity of $E$.

Lemma 2.2. A solution $u \in \mathbf{U}_{\mathbf{a d}}$ of (2.15) solves the variational inequality

$$
\int_{\Omega} \gamma \varepsilon \nabla u \cdot \nabla(\eta-u)-\frac{1}{\varepsilon} \int_{\Omega} u(\eta-u)+\frac{1}{\tau} \int_{\Omega}(-\Delta)^{-1}\left(u-u^{n-1}\right)(\eta-u) \geq 0
$$

for all $\eta \in \mathbf{U}_{\mathbf{a d}}$.

Proof. Computing the first variation of the first two terms in $(2.15)$ in a direction $(\eta-u)$ is standard. In addition we have

$\frac{\mathrm{d}}{\mathrm{d} \delta} \int_{\Omega}\left|\nabla(-\Delta)^{-1}\left(u+\delta(\eta-u)-u^{n-1}\right)\right|_{\mid \delta=0}^{2}=\int_{\Omega} \nabla(-\Delta)^{-1}\left(u-u^{n-1}\right) \cdot \nabla(-\Delta)^{-1}(\eta-u)=\int_{\Omega}(-\Delta)^{-1}\left(u-u^{n-1}\right)(\eta-u)$. 
Since for a minimizer $u$ the derivative of the functional in (2.15) has to be nonnegative in directions $\eta-u$ with $\eta \in \mathbf{U}_{\text {ad }}$ we obtain (2.17).

The following lemma gives the existence of a Lagrange multiplier for the equality constraint ${\underset{\Omega}{\Omega}}_{\mathrm{f}} u=m$.

Lemma 2.3. Let $u \in \mathbf{U}_{\text {ad }}$ be a solution of the variational inequality (2.17). Then there exists a $\lambda \in \mathbb{R}$ such that for all $\eta \in H^{1}(\Omega)$ with $|\eta| \leq 1$ the inequality

$$
\int_{\Omega} \gamma \varepsilon \nabla u \cdot \nabla(\eta-u)-\frac{1}{\varepsilon} \int_{\Omega} u(\eta-u)+\frac{1}{\tau} \int_{\Omega}(-\Delta)^{-1}\left(u-u^{n-1}\right)(\eta-u)-\lambda \int_{\Omega}(\eta-u) \geq 0
$$

holds.

Proof. We argue similar as in the proof of Proposition 3.3 in [6]. Let $f=\frac{2}{\varepsilon} u-\frac{1}{\tau}(-\Delta)^{-1}\left(u-u^{n-1}\right)$. Since $u$ and $u^{n-1}$ are bounded by one in modulus we obtain from the theory of elliptic equations that $f$ is bounded. We now define for each $\alpha \in \mathbb{R}$ a function $u_{\alpha} \in K:=\left\{u \in H^{1}(\Omega)|| u \mid \leq 1\right\}$ such that for all $\eta \in K$

$$
\int_{\Omega} \gamma \varepsilon \nabla u_{\alpha} \cdot \nabla\left(\eta-u_{\alpha}\right)+\frac{1}{\varepsilon} \int_{\Omega} u_{\alpha}\left(\eta-u_{\alpha}\right)-\int_{\Omega} f\left(\eta-u_{\alpha}\right)-\alpha \int_{\Omega}\left(\eta-u_{\alpha}\right) \geq 0 .
$$

Using standard theory of variational inequalities we deduce that (2.19) has a unique solution, see e.g. [33]. We now introduce a function $M: \mathbb{R} \rightarrow \mathbb{R}$ by

$$
M(\alpha):=f_{\Omega} u_{\alpha} .
$$

For all $\eta \in K$ and all $\alpha \in \mathbb{R}$ we have the pointwise inequalities

$$
\begin{gathered}
\left(\frac{1}{\varepsilon}-f-\alpha\right)(\eta-1) \geq\left(\frac{1}{\varepsilon}+\|f\|_{\infty}-\alpha\right)(\eta-1) \\
\text { and } \quad\left(\frac{1}{\varepsilon}-f-\alpha\right)(\eta+1) \geq\left(-\frac{1}{\varepsilon}-\|f\|_{\infty}-\alpha\right)(\eta+1) .
\end{gathered}
$$

Hence, inserting $\eta \equiv \pm 1$ into (2.19) we observe that $u \equiv 1$ is a solution of (2.19) if $\alpha \geq \frac{1}{\varepsilon}+\|f\|_{\infty}$ and $u \equiv-1$ is a solution of (2.19) if $\alpha \leq-\left(\frac{1}{\varepsilon}+\|f\|_{\infty}\right)$. We now obtain $M\left( \pm\left(\frac{1}{\varepsilon}+\|f\|_{\infty}\right)\right)= \pm 1$. As in the proof of Proposition 3.3 in [6] we obtain that $M$ is monotone and continuous. Hence a $\lambda \in \mathbb{R}$ exists such that $M(\lambda)=m$. We now choose $\eta=u_{\lambda}$ in (2.17) and $\eta=u$ in (2.19) where we set $\alpha=\lambda$. Adding both resulting terms leads to

$$
\int_{\Omega} \gamma \varepsilon\left|\nabla\left(u-u_{\lambda}\right)\right|^{2}+\frac{1}{\varepsilon} \int_{\Omega}\left|u_{\lambda}-u\right|^{2} \leq 0,
$$

where we use the fact that $\underset{\Omega}{f} u=f_{\Omega} u_{\lambda}$. Hence $u=u_{\lambda}$. Using this result and the definition of $f$ we conclude from (2.19) that $u$ fulfills (2.18).

Using regularity theory for obstacle problems we obtain similar as in the proof of Lemma 3.2 in [6]

$$
u \in W_{\text {loc }}^{2, p}(\Omega) \text { for all } p \in(1, \infty), \quad u \in C_{\text {loc }}^{1, \alpha}(\Omega) \text { for all } \alpha \in(0,1) .
$$

Setting

$$
\begin{aligned}
v & =-(-\Delta)^{-1}\left(\frac{u-u^{n-1}}{\tau}\right), w=v+\lambda, \\
\mu_{+} & =\varepsilon\left(\gamma \varepsilon \Delta u+\frac{1}{\varepsilon} u+w\right)^{+}=\varepsilon \max \left(\gamma \varepsilon \Delta u+\frac{1}{\varepsilon} u+w, 0\right), \\
\mu_{-} & =\varepsilon\left(\gamma \varepsilon \Delta u+\frac{1}{\varepsilon} u+w\right)^{-}=\varepsilon \max \left(-\gamma \varepsilon \Delta u-\frac{1}{\varepsilon} u-w, 0\right), \\
\mu & =\mu_{+}-\mu_{-}
\end{aligned}
$$

we obtain similar as for other optimization problems with bilateral constraints (see e.g. [43]) result (i) in the following remark. 
Remark 2.1. (i) There exists a solution $(u, v, w, \mu)$ of the KKT system (2.8)-(2.14). In particular there is a Lagrange multiplier $\mu$ for $|u| \leq 1$ with $\mu \in L^{2}(\Omega)$. We also refer to [26,27] for a discrete saddle point formulation related to $(2.8)-(2.14)$.

(ii) Replacing $\psi_{0}^{\prime}(u)$ in $(2.11)$ by $\psi_{0}^{\prime}\left(u^{n-1}\right)$ gives a semi-implicit time discretization, see e.g. [7]. Arguing similar as above a solution to the semi-discrete version exists which is in $H^{2}(\Omega)$. In this case the minimization problem related to (2.15) is always uniquely solvable, i.e. no restriction on the time step is necessary.

\section{Primal-Dual aCtive Set approach}

The goal of this section is to formulate a primal-dual active set method in order to solve for a time step $\tau>0$ a spatially discretized version of

$$
\frac{1}{\tau}\left(u-u^{n-1}\right)=\Delta w \text { in } \Omega, \frac{\partial w}{\partial n}=0 \text { on } \partial \Omega
$$

together with (2.11)-(2.14). We now introduce for a $c>0$ the active sets

$$
A^{+}=\left\{x \in \Omega \mid u(x)+\frac{\mu(x)}{c}>1\right\}, \quad A^{-}=\left\{x \in \Omega \mid u(x)+\frac{\mu(x)}{c}<-1\right\}
$$

and the inactive set $I:=\Omega \backslash\left(A^{+} \cup A^{-}\right)$. The conditions (2.12)-(2.14) can be reformulated as

$$
u(x)= \pm 1 \quad \text { if } x \in A^{ \pm}, \quad \mu(x)=0 \quad \text { if } x \in I .
$$

Formally, this leads to the following primal-dual active set strategy employing the primal variable $u$ and the dual variable $\mu$.

Primal-Dual Active Set Algorithm (PDAS-I):

(1) Set $k=0$, initialize $A_{0}^{ \pm}$and define $I_{0}=\Omega \backslash\left(A_{0}^{+} \cup A_{0}^{-}\right)$.

(2) Set $u_{k}= \pm 1$ on $A_{k}^{ \pm}$and $\mu_{k}=0$ on $I_{k}$.

(3) Solve the coupled system of PDE's (3.1), (2.11) to obtain $u_{k}$ on $I_{k}, \mu_{k}$ on $A_{k}^{+} \cup A_{k}^{-}$and $w_{k}$ on $\Omega$.

(4) Set $A_{k+1}^{+}:=\left\{x \in \Omega \mid u_{k}(x)+\frac{\mu_{k}(x)}{c}>1\right\}$, $A_{k+1}^{-}:=\left\{x \in \Omega \mid u_{k}(x)+\frac{\mu_{k}(x)}{c}<-1\right\}$ and $I_{k+1}=\Omega \backslash\left(A_{k+1}^{+} \cup A_{k+1}^{-}\right)$.

(5) If $A_{k+1}^{ \pm}=A_{k}^{ \pm}$stop, otherwise set $k=k+1$ and goto 2 .

Another reformulation of (2.12)-(2.14) is given with the help of a semi-smooth equation as follows

$$
H(u, \mu):=\mu-(\max (0, \mu+c(u-1))+\min (0, \mu+c(u+1)))=0 .
$$

A semi-smooth Newton method applied in a formal way to (2.11), (3.1), (3.3) is equivalent to the above primaldual active set method, see e.g. [30] for a different context. It is known that for obstacle problems the iterations in (PDAS-I) in general are not applicable in function space since the iterates $\mu_{k}$ are only measures and not $L^{2}$-functions, see [31]. The same is true for the non-standard obstacle problem (2.4), as $\Delta u_{k}$ as obtained in the iterations of (PDAS-I) in general is only a measure. In the following section we introduce a finite element discretization of (2.10)-(2.14) and we show that for the discretized system local convergence holds.

\section{Finite Element Discretization}

We now introduce a finite element approximation for the Cahn-Hilliard variational inequality using continuous, piecewise affine linear finite elements for $u$ and $w$. In the following we assume for simplicity that $\Omega$ is a polyhedral domain. Generalizations to curved domains are possible using boundary finite elements 
with curved faces. Let $\left\{\mathcal{T}_{h}\right\}_{h>0}$ be a triangulation of $\Omega$ into disjoint open simplices. Furthermore, we define $\mathcal{T}_{h}$ to have maximal element size $h:=\max _{T \in \mathcal{T}_{h}}\{\operatorname{diam}(T)\}$ and we set $J_{h}$ to be the set of nodes of $\mathcal{T}_{h}$ and $p_{j} \in J_{h}$ to be the coordinates of these nodes. The finite element space of piecewise affine linear, continuous finite elements associated to $\mathcal{T}_{h}$ is now given as $S_{h}:=\left\{\varphi \in C^{0}(\bar{\Omega}) \mid \varphi_{\left.\right|_{T}} \in P_{1}(T) \quad \forall T \in \mathcal{T}_{h}\right\} \subset H^{1}(\Omega)$ where we denote by $P_{1}(T)$ the set of all affine linear functions on $T$. To each $p_{j} \in J_{h}$ we associate the nodal basis function $\chi_{j} \in S_{h}$ with the property $\chi_{j}\left(p_{i}\right)=\delta_{i j}$. We replace the $L^{2}$-inner product $(.,$.$) at some places by a quadrature rule given$ by the lumped mass inner product $(\eta, \chi)_{h}=\int_{\Omega} I_{h}(\eta \chi)$, where $I_{h}:=C^{0}(\bar{\Omega}) \rightarrow S_{h}$ is the standard interpolation operator at the nodes. In the following, we consider either an implicit or an explicit discretization of the term $\psi_{0}^{\prime}(u)$, i.e. we choose $\psi_{0}^{\prime}\left(u^{*}\right)$ where $* \in\{n-1, n\}$. Then, the spatial discretization of (3.1), (2.11)-(2.14) is given as:

For $n=1,2,3, \ldots$ and given $u_{h}^{0} \in S_{h}$ find iteratively $\left(u_{h}^{n}, w_{h}^{n}, \mu_{h}^{n}\right) \in S_{h} \times S_{h} \times S_{h}$ such that

$$
\begin{array}{cl}
\frac{1}{\tau}\left(u_{h}^{n}-u_{h}^{n-1}, \chi\right)_{h}+\left(\nabla w_{h}^{n}, \nabla \chi\right)=0 & \forall \chi \in S_{h}, \\
\left(w_{h}^{n}, \chi\right)_{h}-\gamma \varepsilon\left(\nabla u_{h}^{n}, \nabla \chi\right)-\frac{1}{\varepsilon}\left(\psi_{0}^{\prime}\left(u_{h}^{*}\right), \chi\right)_{h}-\frac{1}{\varepsilon}\left(\mu_{h}^{n}, \chi\right)_{h}=0 & \forall \chi \in S_{h}, \\
\mu_{h}^{n}=\mu_{h,+}^{n}-\mu_{h,-}^{n}, \quad \mu_{h,+}^{n} \geq 0, \quad \mu_{h,-}^{n} \geq 0, \quad\left|u_{h}^{n}\right| \leq 1, & \\
\mu_{h,+}^{n}\left(p_{j}\right)\left(u_{h}^{n}\left(p_{j}\right)-1\right)=\mu_{h,-}^{n}\left(p_{j}\right)\left(u_{h}^{n}\left(p_{j}\right)+1\right)=0 & \forall p_{j} \in J_{h} .
\end{array}
$$

Notice that (4.4) does in general not imply (2.13) pointwise in all of $\Omega$. Choosing $\chi \equiv 1$ in (4.1) provides the mass conservation ${\underset{\Omega}{\Omega}}_{h} u_{h}^{n}=f_{\Omega} u_{h}^{n-1}=f_{\Omega} u_{h}^{0}$.

The discretization of (2.12)-(2.14) can also be introduced as in Section 3 with the help of active nodes

$$
\mathcal{A}_{h}^{n,+}=\left\{p_{j} \in J_{h} \mid u_{h}^{n}\left(p_{j}\right)+\frac{\mu_{h}^{n}\left(p_{j}\right)}{c}>1\right\}, \mathcal{A}_{h}^{n,-}=\left\{p_{j} \in J_{h} \mid u_{h}^{n}\left(p_{j}\right)+\frac{\mu_{h}^{n}\left(p_{j}\right)}{c}<-1\right\}
$$

for any positive $\mathbf{c}$. Then we define the set of inactive nodes as $\mathcal{I}_{h}^{n}=J_{h} \backslash\left(\mathcal{A}_{h}^{n,+} \cup \mathcal{A}_{h}^{n,-}\right)$ and require

$$
u_{h}^{n}\left(p_{j}\right)= \pm 1 \quad \text { if } p_{j} \in \mathcal{A}_{h}^{n, \pm}, \quad \mu_{h}^{n}\left(p_{j}\right)=0 \quad \text { if } p_{j} \in \mathcal{I}_{h}^{n} .
$$

As discussed in Sections 1 and 2 the equations (4.2), (4.5)-(4.6) can be rewritten as a variational inequality as follows. Introducing the space

$$
K_{h}:=\left\{\eta \in S_{h}|| \eta(x) \mid \leq 1 \quad \text { for all } \quad x \in \Omega\right\}
$$

we search $u_{h}^{n} \in K_{h}$ such that

$$
\left(w_{h}^{n}, \xi-u_{h}^{n}\right)_{h} \geq \gamma \varepsilon\left(\nabla u_{h}^{n}, \nabla\left(\xi-u_{h}^{n}\right)\right)+\frac{1}{\varepsilon}\left(\psi_{0}^{\prime}\left(u_{h}^{n-1}\right), \xi-u_{h}^{n}\right)_{h} \quad \forall \xi \in K_{h} .
$$

In order to compute $\left(u_{h}^{n}, w_{h}^{n}, \mu_{h}^{n}\right)$ we now choose a discretized version of the primal-dual active set method (PDAS-I), where we iteratively update active sets $\mathcal{A}_{h, k}^{n, \pm}$ for $k=0,1,2, \ldots$ We drop for convenience sometimes the indices $n, h$. The following discrete version of the primal-dual active set strategy is obtained using that $\mu_{h}^{n}\left(p_{j}\right)=0$ on $\mathcal{I}_{h, k}^{n}$ in (4.2). Then (4.2) reduces roughly spoken to a discretized PDE for $u_{h}^{n}$ only on an interface given by $\mathcal{I}_{h, k}^{n}$. For known $u_{h}^{n}, w_{h}^{n}(4.2)$ determines $\mu_{h}^{n}$ on the active set. Here one has to use that $(\cdot, \cdot)_{h}$ is a mass lumped $L^{2}$-inner product in order to obtain that in (4.2) unknowns at different nodes only couple through the gradient term, leading to a system splitted according to active and inactive nodes. For the precise formulation we introduce the notation

$$
\tilde{S}_{h, k}:=\left\{\tilde{\chi} \in S_{h} \mid \tilde{\chi}\left(p_{j}\right)=0 \text { if } p_{j} \in \mathcal{A}_{h, k}^{n,+} \cup \mathcal{A}_{h, k}^{n,-}\right\}=\operatorname{span}\left\{\chi_{i} \mid p_{i} \in \mathcal{I}_{h, k}^{n}\right\} .
$$


Primal-Dual Active Set Algorithm (PDAS-II):

(1) Set $k=0$, initialize $\mathcal{A}_{0}^{ \pm}$and define $\mathcal{I}_{0}=J_{h} \backslash\left(\mathcal{A}_{0}^{+} \cup \mathcal{A}_{0}^{-}\right)$.

(2) Solve for $\left(u_{k}, w_{k}\right) \in S_{h} \times S_{h}$ the system

$$
\begin{array}{rlll}
\frac{1}{\tau}\left(u_{k}-u_{h}^{n-1}, \chi\right)_{h}+\left(\nabla w_{k}, \nabla \chi\right) & =0 & \forall \chi \in S_{h}, \\
\left(w_{k}, \tilde{\chi}\right)_{h}-\gamma \varepsilon\left(\nabla u_{k}, \nabla \tilde{\chi}\right)-\frac{1}{\varepsilon}\left(\psi_{0}^{\prime}\left(u_{h}^{*}\right), \tilde{\chi}\right)_{h} & =0 \quad \forall \tilde{\chi} \in \tilde{S}_{h, k}, \\
u_{k}\left(p_{j}\right) & = \pm 1 \quad & \text { if } p_{j} \in \mathcal{A}_{k}^{ \pm} .
\end{array}
$$

(3) Define $\mu_{k} \in S_{h}$ via

$$
\begin{aligned}
\mu_{k}\left(p_{j}\right)\left(1, \chi_{j}\right)_{h} & =\varepsilon\left(w_{k}, \chi_{j}\right)_{h}-\gamma \varepsilon^{2}\left(\nabla u_{k}, \nabla \chi_{j}\right)-\left(\psi_{0}^{\prime}\left(u_{h}^{*}\right), \chi_{j}\right)_{h} \quad \forall p_{j} \notin \mathcal{I}_{k}, \\
\mu_{k}\left(p_{j}\right) & =0 \quad \forall p_{j} \in \mathcal{I}_{k} .
\end{aligned}
$$

(4) Set $\mathcal{A}_{k+1}^{+}:=\left\{p_{j} \in J_{h} \mid u_{k}\left(p_{j}\right)+\frac{\mu_{k}\left(p_{j}\right)}{c}>1\right\}$,

$$
\mathcal{A}_{k+1}^{-}:=\left\{p_{j} \in J_{h} \mid u_{k}\left(p_{j}\right)+\frac{\mu_{k}\left(p_{j}\right)}{c}<-1\right\} \text { and } \mathcal{I}_{k+1}=J_{h} \backslash\left(\mathcal{A}_{k+1}^{+} \cup \mathcal{A}_{k+1}^{-}\right) .
$$

(5) If $\mathcal{A}_{k+1}^{ \pm}=\mathcal{A}_{k}^{ \pm}$stop, otherwise set $k=k+1$ and goto 2 .

Lemma 4.1. For all $u_{h}^{n-1} \in S_{h}$ and $\mathcal{A}_{k}^{ \pm}$there exists a unique solution $\left(u_{k}, w_{k}\right) \in S_{h} \times S_{h}$ of (4.8)-(4.10) with $*=(n-1)$, i.e. the semi-implicit case, provided that $\mathcal{I}_{k}=J_{h} \backslash\left(\mathcal{A}_{k}^{+} \cup \mathcal{A}_{k}^{+}\right) \neq \emptyset$.

Proof. The idea of this proof is to consider the discretized version of (2.4) and (2.5) under the constraint $u= \pm 1$ on $\mathcal{A}_{k}$ and to use ideas similar to the existence proof in Lemma 2.1. Hence, we define $S_{h, m}:=\left\{\chi \in S_{h} \mid\right.$ $\underset{\Omega}{f} \chi=m\}$, where $m:=f_{\Omega} u_{h}^{n-1}$

$$
S_{h}^{I}:=\left\{u \in S_{h} \mid u\left(p_{j}\right)=1 \text { if } j \in \mathcal{A}_{k}^{+}, u\left(p_{j}\right)=-1 \text { if } j \in \mathcal{A}_{k}^{-}\right\},
$$

and $S_{h, m}^{I}:=S_{h}^{I} \cap S_{h, m}$. Since $\mathcal{I}_{k} \neq \emptyset$ we conclude $S_{h, m}^{I} \neq \emptyset$. The discrete inverse Laplacian $\left(-\Delta_{h}\right)^{-1}: S_{h, 0} \rightarrow$ $S_{h, 0}, \eta^{h} \mapsto\left(-\Delta_{h}\right)^{-1} \eta^{h}$ is defined via

$$
\left(\nabla\left(\left(-\Delta_{h}\right)^{-1} \eta^{h}\right), \nabla \chi\right)=\left(\eta^{h}, \chi\right)_{h} \quad \text { for all } \chi \in S_{h, 0} .
$$

Since the homogeneous problem only has the trivial solution and $S_{h, 0}$ is finite dimensional, the linear equation (4.15) has a unique solution. We define $u_{k} \in S_{h, m}^{I}$ as the solution of the minimization problem

$$
\min _{\eta \in S_{h, m}^{I}}\left\{\frac{1}{2 \tau}\left(\nabla\left(-\Delta_{h}\right)^{-1}\left(\eta-u_{h}^{n-1}\right), \nabla\left(-\Delta_{h}\right)^{-1}\left(\eta-u_{h}^{n-1}\right)\right)+\frac{\gamma \varepsilon}{2}(\nabla \eta, \nabla \eta)+\frac{1}{\varepsilon}\left(\psi_{0}^{\prime}\left(u_{h}^{n-1}\right), \eta\right)_{h}\right\}
$$

which exists uniquely since the Poincaré inequality similar as in the proof of Lemma 2.1 implies coerciveness. Computing the first variation of the minimisation problem (4.16) gives for the solution $u_{k} \in S_{h, m}^{I}$

$$
0=\frac{1}{\tau}\left(\nabla\left(-\Delta_{h}\right)^{-1}\left(u_{k}-u_{h}^{n-1}\right), \nabla\left(-\Delta_{h}\right)^{-1} \tilde{\chi}\right)+\gamma \varepsilon\left(\nabla u_{k}, \nabla \tilde{\chi}\right)+\frac{1}{\varepsilon}\left(\psi_{0}^{\prime}\left(u_{h}^{n-1}\right), \tilde{\chi}\right)_{h}
$$

for all $\tilde{\chi} \in \tilde{S}_{h, k}$ with $f_{\Omega} \tilde{\chi}=0$. Now we define $w_{k} \in S_{h}$ as

$$
w_{k}=-\left(-\Delta_{h}\right)^{-1}\left(\frac{u_{k}-u_{h}^{n-1}}{\tau}\right)+\lambda_{k}
$$

where $\lambda_{k} \in \mathbb{R}$ is uniquely given with the help of any nodal basis function $\chi_{j} \in S_{h}$ with $p_{j} \in \mathcal{I}_{k}$ by

$$
\lambda_{k}=\left\{\frac{1}{\tau}\left(\left(-\Delta_{h}\right)^{-1}\left(u_{k}-u_{h}^{n-1}\right), \chi_{j}\right)_{h}+\gamma \varepsilon\left(\nabla u_{k}, \nabla \chi_{j}\right)+\frac{1}{\varepsilon}\left(\psi_{0}^{\prime}\left(u_{h}^{n-1}\right), \chi_{j}\right)\right\} /\left(1, \chi_{j}\right) .
$$


Using the definition of the discrete inverse Laplacian, see (4.15), and the fact that $\underset{\Omega}{f} u_{k}=\underset{\Omega}{f} u^{n-1}$ now gives that (4.8) holds. Furthermore (4.17), (4.15) and (4.18) imply that (4.9) holds for all $\tilde{\chi} \in \tilde{S}_{h, k}$ with $\underset{\Omega}{f} \tilde{\chi}=0$. For $\tilde{\chi} \in \tilde{S}_{h, k}$ which do not satisfy the integral constraint $f_{\Omega} \tilde{\chi}=0$ we set $\hat{\chi}:=\tilde{\chi}-\alpha \chi_{j}$ with $p_{j} \in I_{k}$ and $\alpha \in \mathbb{R}$ such that $\int_{\Omega} \hat{\chi}=0$. With this choice of $\hat{\chi}$ as a test function in (4.17) we can conclude with the help of (4.15), (4.18) and (4.19) that (4.9) holds for all $\tilde{\chi} \in \tilde{S}_{h, k}$. Hence (4.8)-(4.10) has a solution.

It remains to prove uniqueness. Let us assume that (4.8)-(4.10) has two solutions $\left(u_{k, 1}, w_{k, 1}\right),\left(u_{k, 2}, w_{k, 2}\right) \in$ $S_{h} \times S_{h}$. Then we obtain for the differences $v=u_{k, 1}-u_{k, 2}, z=w_{k, 1}-w_{k, 2}$ by testing (4.8), (4.9) for $\left(u_{k, 1}, w_{k, 2}\right)$ and $\left(u_{k, 2}, w_{k, 2}\right)$ with $v$ and $z$ respectively, after taking differences:

$$
(v, z)_{h}+\tau\|\nabla z\|_{L^{2}}^{2}-(z, v)_{h}+\gamma \varepsilon\|\nabla v\|_{L^{2}}^{2}=0 .
$$

Since $\underset{\Omega}{f} u_{k, 1}=f_{\Omega} u_{k, 2}=\underset{\Omega}{f} u^{n-1}$ we obtain $v \equiv 0$ in $\Omega$ and hence $u_{k, 1}=u_{k, 2}$. The identities (4.8), (4.9) imply that necessarily the identities (4.18) and (4.19) have to hold. This implies that also $w_{k}$ is unique. We remark that this uniqueness result also implies that the definition of $\lambda_{k}$ in (4.19) does not depend on $j$.

Now $\mu_{k}$ is uniquely defined by (4.11), (4.12) and hence taking Lemma 4.1 into account we obtain that a unique solution of (4.8)-(4.12) exists.

In the following we require the condition $\mathcal{I}_{k}=J_{h} \backslash\left(\mathcal{A}_{k}^{+} \cup \mathcal{A}_{k}^{-}\right) \neq \emptyset$, which guarantees that there is a $u \in S_{h}$ such that $\underset{\Omega}{f} u=m$. Otherwise (4.8) is not solvable.

Remark 4.1. In order to solve (4.8)-(4.12) the main computational effort is to solve the system (4.8), (4.9) which has a specific structure. The discretized elliptic equation (4.8) for $w$ is defined on the whole of $\Omega$ whereas the elliptic equation (4.9) is defined only on the inactive set, see Figure 1. The two equations are coupled in a way which leads to an overall symmetric system which will be used later when we propose numerical algorithms.

The discretization of (2.12)-(2.14) can also be formulated with the help of the semi-smooth function $H$, see (3.3), as a nonlinear equation

$$
H\left(u_{h}^{n}\left(p_{j}\right), \mu_{h}^{n}\left(p_{j}\right)\right)=0 \quad \forall p_{j} \in J_{h} .
$$

The formulation (4.1), (4.2), (4.20) was the basis of a numerical method introduced by Blowey and Elliott [7]. In [7] a splitting method is used where the equations (4.1) and (4.2) are used to update approximations of $\left(u_{h}^{n}, w_{h}^{n}\right)$ and in a second step a recursive iteration of (4.20) is used to update the approximation of $\mu_{h}^{n}$.

Using the approach of [30] we can interpret (PDAS-II) as a semi-smooth Newton method for the system (4.1), (4.2), (4.20) and we obtain the following local convergence result for the semi-implicit discretization.

Theorem 4.1. Let $(u, w, \mu) \in S_{h} \times S_{h} \times S_{h}$ be a solution of (4.1), (4.2), (4.20) with * $=(n-1)$ such that $\left\{p_{j} \in J_{h}|| u\left(p_{j}\right) \mid<1\right\} \neq \emptyset$. Then the semi-smooth Newton method for (4.1), (4.2), (4.20) and hence (PDAS-II) converges in a neighbourhood of $(u, w, \mu)$.

Proof. Showing the existence of a solution to (4.1), (4.2), (4.20) is equivalent to the problem of finding a zero of the mapping

$$
G: S_{h} \times S_{h} \times S_{h} \rightarrow S_{h} \times S_{h} \times S_{h}
$$

where for $(u, w, \mu) \in S_{h} \times S_{h} \times S_{h}$ we define $G=\left(G_{1}, G_{2}, G_{3}\right)$ via

$$
\begin{aligned}
\left(G_{1}(u, w, \mu), \chi\right)_{h} & :=\left(u-u_{h}^{n-1}, \chi\right)_{h}+\tau(\nabla w, \nabla \chi), \\
\left(G_{2}(u, w, \mu), \chi\right)_{h} & :=(w, \chi)_{h}-\gamma \varepsilon(\nabla u, \nabla \chi)-\frac{1}{\varepsilon}\left(\psi_{0}^{\prime}\left(u_{h}^{n-1}\right), \chi\right)_{h}-\frac{1}{\varepsilon}(\mu, \chi)_{h}, \\
\left(G_{3}(u, w, \mu), \chi\right)_{h} & :=(H(u, \mu), \chi)_{h} .
\end{aligned}
$$


The min-max-function $H(u, \mu)$, see (3.3), is slantly differentiable and a slanting function is given by $D H(u, \mu)=(0,1)$ if $\left|u+\frac{\mu}{c}\right| \leq 1$ and $D H(u, \mu)=(-c, 0)$ otherwise (see [27]). As a consequence $G$ is slantly differentiable. Moreover similar as in [27] we can derive that the primal-dual active set method (PDAS-II) is equivalent to a semi-smooth Newton method for $G$. We now get local convergence of (PDAS-II) if we can show that the slanting function of $G$ is invertible in a neighbourhood of $(u, w, \mu)$ and the inverses are uniformly bounded $[13,30]$.

The semi-smooth derivative (slanting function) of $G$ is invertible at $(\hat{u}, \hat{w}, \hat{\mu}) \in S_{h} \times S_{h} \times S_{h}$ if and only if we can show injectivity, i.e. that a unique solution $(\bar{u}, \bar{w}, \bar{\mu}) \in S_{h} \times S_{h} \times S_{h}$ of the following linear system exists

$$
\begin{gathered}
(\bar{u}, \chi)_{h}+\tau(\nabla \bar{w}, \nabla \chi)=0, \forall \chi \in S_{h}, \\
(\bar{w}, \chi)_{h}-\gamma \varepsilon(\nabla \bar{u}, \nabla \chi)-\frac{1}{\varepsilon}(\bar{\mu}, \chi)_{h}=0, \forall \chi \in S_{h}, \\
\bar{u}\left(p_{j}\right)=0 \quad \text { if } \quad p_{j} \in \hat{\mathcal{A}}:=\left\{p_{j} \in J_{h}|| \hat{u}\left(p_{j}\right)+\frac{\hat{\mu}\left(p_{j}\right)}{c} \mid>1\right\}, \\
\bar{\mu}\left(p_{j}\right)=0 \quad \text { if } \quad p_{j} \in \hat{\mathcal{I}}:=J \backslash \hat{\mathcal{A}} .
\end{gathered}
$$

Testing (4.21) with $\bar{w},(4.22)$ with $\bar{u}$ and using $(\bar{\mu}, \bar{u})_{h}=0$ we obtain

$$
\tau(\nabla \bar{w}, \nabla \bar{w})+\gamma \varepsilon(\nabla \bar{u}, \nabla \bar{u})=0 .
$$

This implies that $\bar{u}$ and $\bar{w}$ are constant. Then (4.21) gives $\bar{u} \equiv 0$. Using the fact that there exists a $p_{j} \in J_{h}$ with $\left|u\left(p_{j}\right)\right|<1$ and $\mu\left(p_{j}\right)=0$ we can guarantee that $\left|\hat{u}\left(p_{j}\right)+\frac{\hat{\mu}\left(p_{j}\right)}{c}\right| \leq 1$ for $(\hat{u}, \hat{w}, \hat{\mu})$ in a ball around $(u, w, \mu)$. Hence, $\hat{\mathcal{I}} \neq \emptyset$ for $(\hat{u}, \hat{w}, \hat{\mu})$ out of this neighbourhood. Testing in (4.22) with $\chi_{j}$ where $p_{j} \in J_{h}$ implies $\bar{w} \equiv 0$ and finally (4.24) and (4.22) yield $\bar{\mu} \equiv 0$.

The semi-smooth derivatives only differ if the active and inactive sets change. Since only a finite number of different choices of these sets are possible we obtain that the inverses are uniformly bounded for all $(\hat{u}, \hat{w}, \hat{\mu})$ with a non-vanishing inactive set $\hat{\mathcal{I}}$. Since we can find an open neighbourhood of $(u, w, \mu)$, where the condition $\hat{\mathcal{I}} \neq \emptyset$ holds, we proved the theorem.

Remark 4.2. Let $(u, w, \mu)$ be a solution to (4.1), (4.2), (4.20). The proof of Theorem 4.1 requires a neighbourhood of $(u, w, \mu)$, where the active sets do not vanish. This can limit the size of the neighbourhood in which local convergence can be guaranteed. However in numerical simulations the mesh size always has to be chosen such that at least eight points lie across the interface. Hence the above mentioned condition never led to any problems in practice.

Remark 4.3. Theorem 4.1 holds also for the implicit discretization if $\tau<4 \gamma \varepsilon^{3}$.

Proof. The proof follows along the lines of the proof of Theorem4.1 if one can show injectivity. Equation (4.22) changes to

$$
(\bar{w}, \chi)_{h}-\gamma \varepsilon(\nabla \bar{u}, \nabla \chi)-\frac{1}{\varepsilon}(\bar{\mu}, \chi)_{h}+\frac{1}{\varepsilon}(\bar{u}, \chi)_{h}=0 .
$$

The same testing as above leads to $\tau(\nabla \bar{w}, \nabla \bar{w})+\gamma \varepsilon(\nabla \bar{u}, \nabla \bar{u})-\frac{1}{\varepsilon}(\bar{u}, \bar{u})_{h}=0$ and testing (4.21) with $\bar{u}$ yields $(\bar{u}, \bar{u})_{h}=\tau(\nabla \bar{w}, \nabla \bar{u})$. Then, if $\tau<4 \gamma \varepsilon^{3}$ we obtain with Young's inequality $\nabla \bar{w}=\nabla \bar{u}=0$. We can now argue as in the proof of Theorem 4.1 that $\bar{u}=\bar{w}=0$ which implies injectivity.

Remark 4.4. The assertion of local convergence in Theorem 4.1 is limited as it is for any local convergence result for finite dimensional PDAS-methods, see e.g. [30], where a nonlinear behavior only enters through the inequality constraints. Local exactness, i.e. convergence in one step, is guaranteed by Lemma 4.1 if $\mathcal{A}_{k}^{ \pm}=\mathcal{A}_{h}^{n, \pm}$. However, we presented the interpretation as a semi-smooth Newton method and its local convergence mainly to demonstrate that the results in [30] are applicable to the fully discretized Cahn-Hilliard variational problem as far as bilateral bounds are not an obstruction. In addition, the arguments can be used for a convergence proof for problems with further nonlinearities for example more complex potentials. Then convergence in finite steps is not guaranteed but a superlinear convergence takes place. 


\section{NUMERICAL RESULTS}

In this section we discuss four test examples and numerically analyse the behaviour of the PDAS-algorithm. In the first test example we consider two concentric circles, where the exact sharp interface solution is known, and compare semi-implicit and implicit discretization.

The second example is a four-circle problem where concave as well as convex sections appear in the interface. With this example we compare the PDAS-method with a standard solver for the Cahn-Hilliard inequality, namely with a projected block Gauss-Seidel method [4]. It turns out that the PDAS-method is more efficient and reliable. In particular, the speed up can be gained by a linear algebra solver which is not based on a GaussSeidel method. Moreover, we have not seen a difference in CPU-time between the semi-implicit and implicit discretization, and in the latter case have not faced such a severe restriction on the time step $\tau$ as indicated by the analysis. The number of PDAS-iterations for each time step were rather depending on $\tau$ than on the mesh size $h$, never exceeded 11 and after the interface settles 2-4 iterations were sufficient. For the number of iterations there was nearly no difference between an adaptive and a uniform grid. But of course in CPU-time adaptivity was much more efficient. In the third test example we considered random initial data, i.e. a starting situation without pure phases. Here and in our last example, a 3D simulation, we observed that even with large topological changes the maximum number of PDAS-iterations stayed always below 10.

Before we present the examples we discuss some numerical issues as there are mesh generation, adaptivity in order to resolve the interface, choice of the parameter $c$, initialization of the active sets and the linear algebra solver. Also we describe shortly the mentioned projected Gauss-Seidel type algorithm.

\section{Mesh generation and adaptivity}

For all the simulations presented in this paper the finite element toolbox ALBERTA [40] was used for mesh generation, the assembly of the matrices and administration. To generate the adaptive meshes we used the mesh adaption strategy of Barrett et al. [4]. Experiments showed that it is essential to ensure that at least eighth vertices are present on the interfaces to avoid mesh effects, see also [8]. We hence refine on the interface down to a level where eight vertices are present and coarse in the areas where the concentration $u$ is constant. For given parameters $\varepsilon$ and $\gamma$ this results in an upper bound $h_{\text {fine }} \leq \varepsilon \sqrt{\gamma} \frac{\pi}{9}$, where $h_{\text {fine }}$ is the refinement level on the interface. We remark here that for the potential $\psi_{0}$ in (1.4) the interfacial thickness is $\varepsilon \sqrt{\gamma} \pi$, compare [8]. Since we want to avoid too coarse meshes we additionally define $h_{\text {coarse }}:=10 \cdot h_{\text {fine }}$ and choose a tolerance tol $=10^{-6}$. Afterwards the mesh adaption is done the following way: For each element $T \in \mathcal{T}^{h}$ calculate the indicator $\eta_{T}:=\left|\min _{x \in T}\right| u(x)|-1|$. Then, a triangle is marked for refinement if it, or one of its neighboring elements,

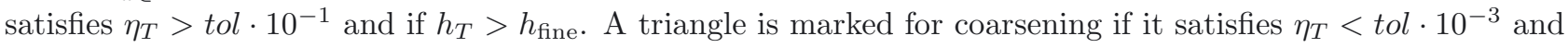
$h_{T}<h_{\text {coarse }}$.

\section{Choice of the parameter $c$}

To determine the active sets we have to choose the parameter $c>0$. In the unilateral case the selection of $c>0$ has no influence on the iterates after the first iteration and can be chosen arbitrary, see [30]. However this is no longer true in the case of bilateral bounds. This is discussed for obstacle problems in [5]. If $c$ is chosen too small we observed cases in which the iterates oscillated and the algorithm did not converge. Figure 2 shows the values of $u$ at various PDAS iterations in one time step of a simulation in one space dimension with $h=\frac{1}{512}$, $\tau=10^{-5}, \pi \varepsilon=0.2$ and $c=0.01$. In the eighth iteration the algorithm breaks down because all vertices are in the active set and the system no longer exhibits a valid solution, compare Remark 4.2. Redoing the simulation with $c=0.2$ fixed the problem and after two iterations the time step was completed with only marginal changes to $u$ since the initial data was close to a stationary solution. The same phenomenon was observed in higher space dimensions.

A heuristic approach showed that it is sufficient to ensure that no vertex can change from the positive active set $\mathcal{A}^{+}$to the negative inactive set $\mathcal{A}^{-}$and vice versa in one iteration. This can be achieved by selecting a PDAS parameter c large enough, depending on the magnitude of the Lagrange multiplier $\mu$. 


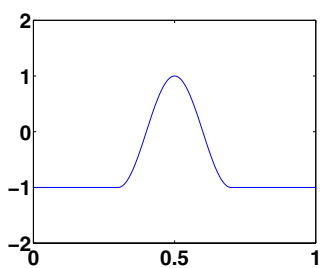

(a) $u_{h}^{0}$

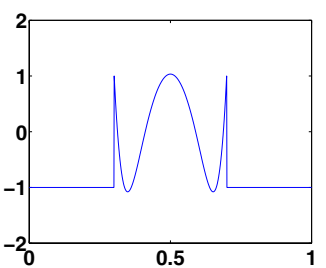

(b) $u_{h}^{3}$

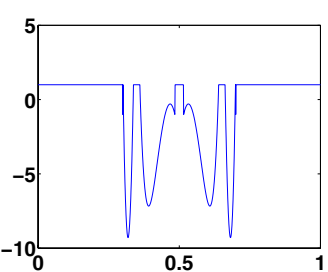

(c) $u_{h}^{5}$

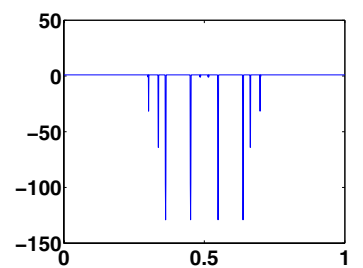

(d) $u_{h}^{7}$

Figure 2. Oscillations in 1D if $c$ is too small.

In all the simulations a value of $c=10$ was sufficient when the interfaces were already well developed and adequate initial guesses for the active sets were known. Therefore, if not mentioned otherwise $c=10$ is chosen in the calculation. In simulations with distortions or jumps in the concentration $u$ larger values depending on the mesh size were necessary. Choosing the parameter $c$ larger had no discernible influence on the simulation.

When using adaptive meshes for the PDAS algorithm a choice has to be made for every newly created vertex if it should belong to the active or inactive set. For now we restrict ourselves to the following: If all neighboring vertices are active then this vertex should be active too. In every other case we set the new vertex to inactive.

\section{Initialization of active sets}

As mentioned previously the application of a PDAS-method to the interface evolution has the advantage that the good initialization due to the information from the previous time step leads to a large speedup. At the first time step $n=1$ the active set $\mathcal{A}_{0}^{n, \pm}$ is initialized using the given initial data $u_{h}^{0}$. Since in the limit the active sets describe the sets where $u$ is strictly active a good approximation of $\mathcal{A}_{0}^{1, \pm}$ is given by the active set of $u_{h}^{0}$. Hence we choose $\mathcal{A}_{0}^{1, \pm}=\left\{p_{j} \in S^{h}|| u_{h}^{0}\left(p_{j}\right) \mp 1 \mid \leq 10^{-8}\right\}$.

For time steps $n \geq 2$ we can exploit in addition $\mu_{h_{n-1}}^{n-1}$. Due to possible grid changes from time step $n-1$ to time step $n$ one may have to apply additionally the standard interpolation $I_{h_{n}}$ to the new grid $S_{h_{n}}$, i.e. with $u_{-1}:=I_{h_{n}} u_{h_{n-1}-1}^{n-1}$ and $\mu_{-1}:=I_{h_{n}} \mu_{h_{n-1}}^{n-1}$ initialize the active set $\mathcal{A}_{0}^{n, \pm}$ as in (4.13) and (4.14). However a less time consuming way is to initialize the active set in the following way, which is applied in this paper: if an edge between two positive or two negative active vertices is bisected, the new vertex is set active and otherwise the new vertex is set inactive.

Solver for the system (4.8)-(4.9)

For moderate mesh sizes direct solvers for sparse linear systems perform quite well. We use a Cholesky decomposition of the system by means of the multifrontal method by Duff and Reid [18] which is realized in the software package UMFpack $[15,16]$. This method is a generalization to the frontal method of Irons [32]. One crucial point of this method is that the decomposition steps of the matrix are computed but not directly applied to the system matrix. Furthermore an elimination tree and pivoting strategy are used which makes use of the sparsity of the system. For further discussion of the method we refer to $[17,36]$. We solved the whole reduced symmetric sparse system (4.8)-(4.9) with (4.10) by UMFpack all at once.

Up to now in our numerical experiments this direct solver performed better than e.g. a block SOR method. The application of other methods like, for example a cg-method for a preconditioned Schur-Complement or block multigrid method, are currently under investigation. In the first tests, which were of moderate size, UMFpack was still faster. The limit of the UMFpack up to now was the available memory. However large 3D problems have not been investigated thoroughly yet.

Gauss-Seidel type algorithm for the variational inequality ( $p S O R$ )

The following Gauss-Seidel type algorithm is often used for solving the Cahn-Hilliard variational inequality, see [22], and is implemented in the same hardware and software environment as our method. Hence to obtain 
a comparison within the same setting we use this method as a reference method. This method is based upon the discretization of the variational inequality (1.16)-(1.17) by a semi-implicit backwards Euler method in time and by using continuous piecewise linear elements in space, compare [7]. This results in the same discretization as we introduced in this paper, namely (4.1) and (4.7):

For $n=1,2,3, \ldots$ and given $u_{h}^{0} \in K_{h}$ find $\left(u_{h}^{n}, w_{h}^{n}\right) \in K_{h} \times S_{h}$ such that

$$
\begin{aligned}
\frac{1}{\tau}\left(u_{h}^{n}-u_{h}^{n-1}, \chi\right)_{h}+\left(\nabla w_{h}^{n}, \nabla \chi\right)=0 & \forall \chi \in S_{h}, \\
\gamma \varepsilon\left(\nabla u_{h}^{n}, \nabla\left(\xi-u_{h}^{n}\right)\right)-\left(w_{h}^{n}, \xi-u_{h}^{n}\right)_{h}+\frac{1}{\varepsilon}\left(\psi_{0}^{\prime}\left(u_{h}^{n-1}\right), \xi-u_{h}^{n}\right)_{h} \geq 0 & \forall \xi \in K_{h} .
\end{aligned}
$$

As is known for obstacle problems (see e.g. [22]) one can apply for this nonstandard variational inequality a projected block-Gauss-Seidel or a projected block-SOR method (pSOR). This has been studied in [4], where also convergence has been shown. The blocks are determined by the $2 \times 2$ blocks corresponding to the values $\left(u_{h}^{n}\left(p_{j}\right), w_{h}^{n}\left(p_{j}\right)\right)$, which are merged for each vertex. In our numerical experiments we use the pSOR-method with overrelaxation using $\omega=1.3$ for comparison with the PDAS-method. As stopping criteria $\left\|u_{k}-u_{k-1}\right\|_{2} \leq 10^{-7}$ and a maximum of 50000 iterations is used.

\section{Splitting approach solver by Blowey and Elliott}

The system (4.1)-(4.2) in conjunction with the semi-smooth equation (4.20) has been previously considered in [7], as mentioned in Section 4. The resulting linear system is stated in (4.14) of [7]. The matrix is independent of the iteration step in contrast to the PDAS-method considered in this paper. The authors in [7] solve this system with a discrete cosine transformation and hence rely on an equidistant discretization.

We implemented the method using the direct solver UMF-Pack to solve (4.14) [7] with an adaptive grid. The number of iterations needed in each time step is considerably larger than the amount of PDAS iterations needed. See Table 2 in the forthcoming Example 2.

\section{Test cases}

\section{Example 1: two concentric circles}

The distinction we made in Section 4 between the explicit and implicit discretization of the free energy term leads to differences in the accuracy of the resulting schemes. We use a radial symmetric situation where the exact sharp interface solution is known to compare these two schemes. The initial data are such that the interface is described by two concentric circles with radii $0<r_{1}(0)=0.05<r_{2}(0)=0.15<1$. They both will shrink over time - the smaller faster than the larger circle - until the smaller one vanishes. Then the solution remains stable due to mass conservation.

In the limit $\varepsilon \rightarrow 0$ the Cahn-Hilliard model describes the evolution of the sharp interface Mullins-Sekerka model, see $[8,39]$. The radial symmetric sharp interface solution is discussed in $[12,41]$. In the above situation the exact sharp interface solution can be calculated as solution of an ODE

$$
r_{1}^{\prime}=-\frac{1}{r_{1}} \frac{\sigma}{r_{1} r_{2}} \frac{r_{1}+r_{2}}{\ln \left(r_{1}\right)-\ln \left(r_{2}\right)}, \quad r_{2}^{\prime}=-\frac{1}{r_{2}} \frac{\sigma}{r_{1} r_{2}} \frac{r_{1}+r_{2}}{\ln \left(r_{1}\right)-\ln \left(r_{2}\right)}
$$

where $\sigma=\frac{\pi}{8}$. For the comparison between the semi-implicit and implicit discretization we used an equidistant mesh.

In Figure 3 we plotted the sum of both radii for fixed $\varepsilon=0.0039$ and varying time step width $\tau$. In the implicit case we can choose even larger $\tau$ and already gain a good approximation of the evolution unlike for the semi-implicit case where a smaller time step is necessary to achieve the desired result. This has been also observed when considering the convergence with respect to $\varepsilon$ to the sharp interface with a fixed time step. Also here the time step can be chosen larger in the implicit case. In the implicit case the curves for $\tau=10^{-6}$ and $\tau=10^{-7}$ nearly coincide. Hence the smaller time step size provides no advantage. The remaining error can only be reduced by scaling down $\varepsilon$. 


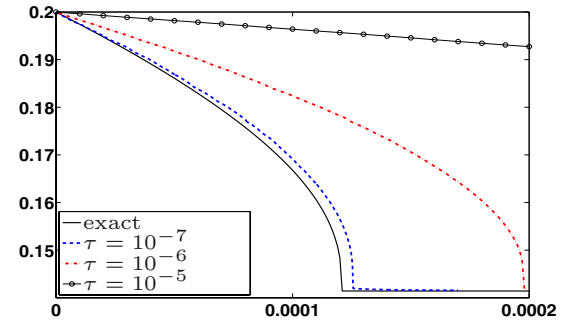

(a) Semi-implicit

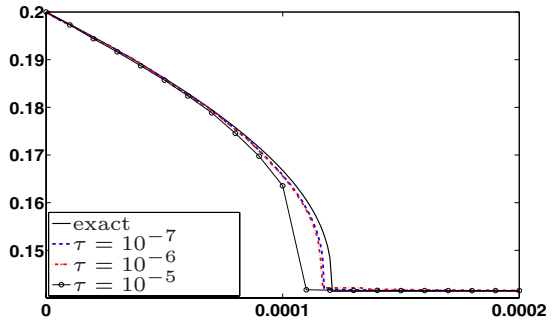

(b) Implicit

Figure 3. $\left(r_{1}+r_{2}\right)(t)$ for different $\tau$ with $\varepsilon=0.0039$.

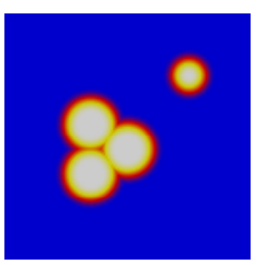

(a) $u_{h}^{0}$

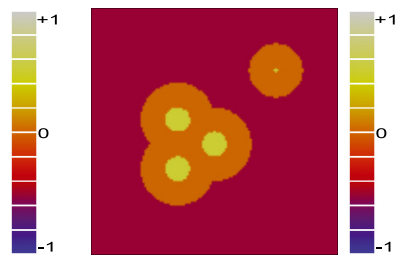

(b) $\mathcal{A}_{h}^{0, \pm}, \mathcal{I}_{h}^{0}$

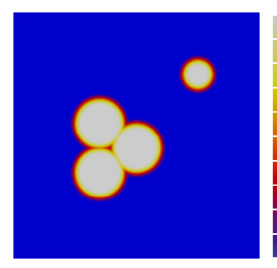

(c) $u_{h}^{0}$

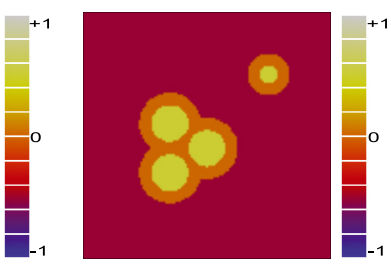

(d) $\mathcal{A}_{h}^{0, \pm}, \mathcal{I}_{h}^{0}$

Figure 4. Initial data for $\pi \varepsilon=0.1$ (left) and $\pi \varepsilon=0.05$ (right).

\section{Example 2: 4-circles problem}

In this example we choose initial data with a concave as well as a convex section of the interface. The initial data on $\Omega=(0,1)^{2}$ consist of four circular interfaces of width $\varepsilon \pi$. The centers and radii are chosen in such a way that three of circles intersect and one is detached. The values \pm 1 are connected by a sine profile which is given as the lowest order term in an asymptotic expansion of the Cahn-Hilliard variational inequality, see e.g. [8]. In Figure 4 we show the initial data for two different interface width. The initial active sets show a value of 0 on each inactive vertex and a positive resp. negative value on each active vertex.

We set $T_{\text {end }}=0.02$ for all the following simulations. In Figures 5 and 6 the evolution of $u, w$ and $\mu$ in time is plotted. Here we used a semi-implicit discretization with an adaptive mesh with $h_{\text {fine }}=0.01$ for $\pi \varepsilon=0.1$ and $h_{\text {fine }}=0.005$ for $\pi \varepsilon=0.05$ respectively, the time step $\tau=10^{-5}$. Simulations with equidistant mesh give the same results. The columns from left to right show the values of $u, w, \mu$ and the mesh after 5, 50, 100 and 200 time steps.

Table 1 shows that for a small number of vertices the pSOR algorithm is still fast but with an increasing number of vertices its performance quickly deteriorates. Using the corresponding block SOR-method in combination with the PDAS-method, the resulting solver is even a bit slower for large time steps. The direct solver on the other hand lowers the runtime considerably. Moreover we see that there is nearly no difference in CPU-time between the semi-implicit and the implicit discretization. The severe restriction on the time step for the implicit case as stated in Lemma 2.1 has not been observed. Only for $\pi \varepsilon=0.05$ the choice $\tau=10^{-4}$ failed even for very large parameter $c=10^{10}$.

When we compare the runtimes used on the fixed mesh with 16641 vertices we notice that the simulations with $\pi \varepsilon=0.2$ used up almost double the time of the one with $\pi \varepsilon=0.1$. The reason lies in the size of the inactive set, which is roughly spoken the interface with width $\pi \varepsilon$. Hence for $\pi \varepsilon=0.2$ the system (4.8)-(4.9) which has to be solved is of larger dimension. 
$u$

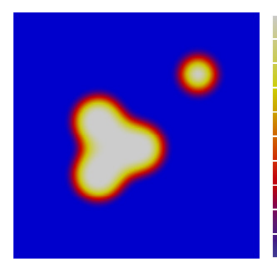

$w$
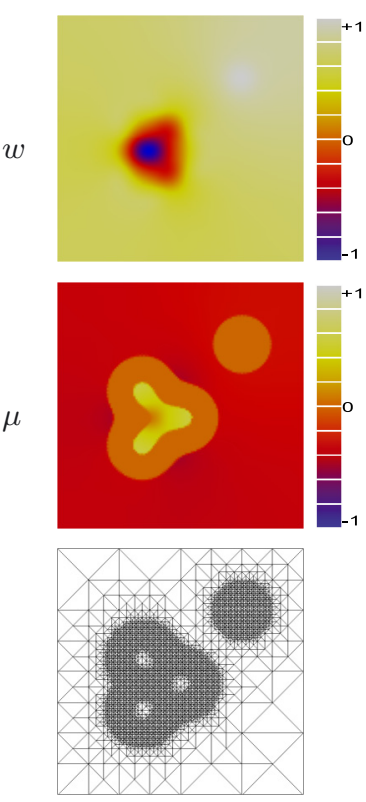

(q) $\mathrm{n}=5$
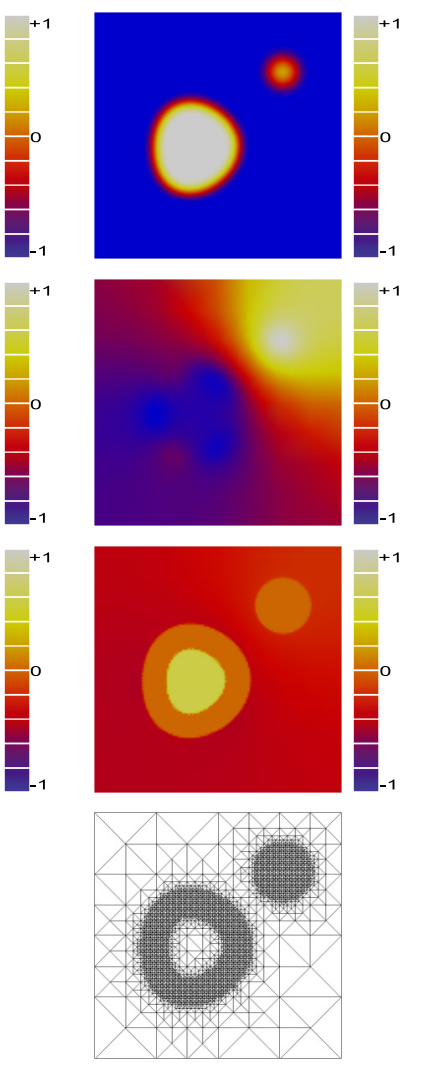

(r) $\mathrm{n}=50$
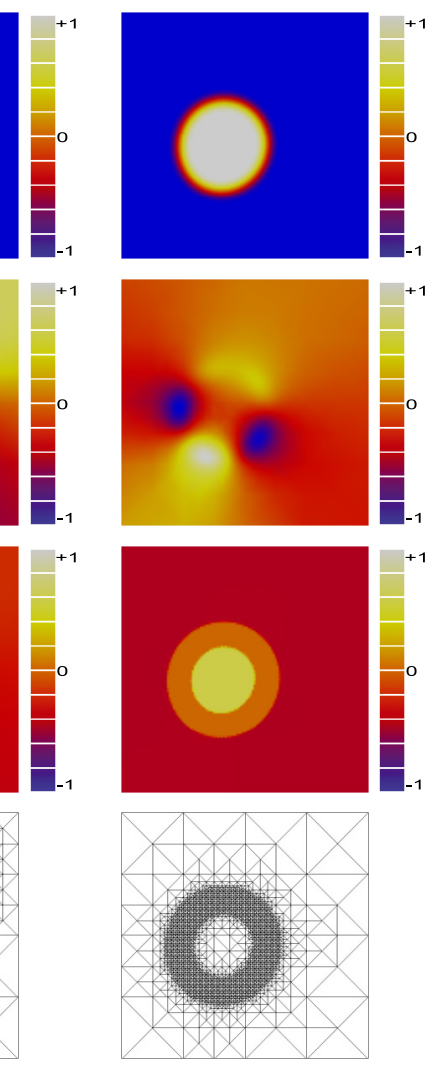

(s) $\mathrm{n}=100$
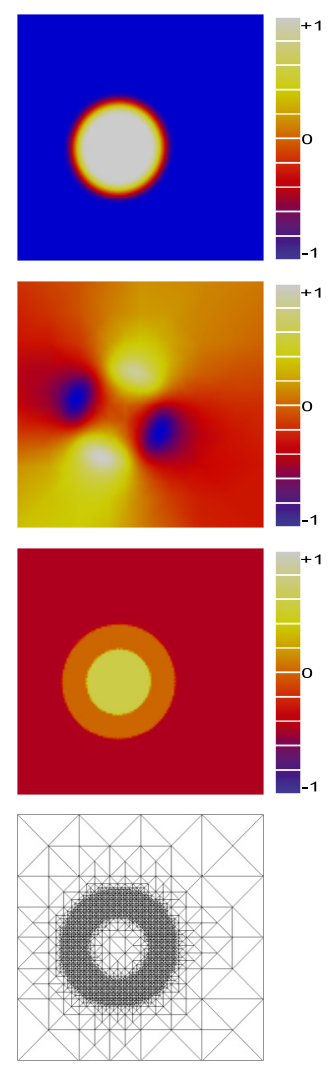

(t) $\mathrm{n}=200$

Figure 5. Time evolution of Example 2 with $\pi \varepsilon=0.1$.

In addition in Table 1 the total, the averaged and the maximal number of PDAS-iterations are listed for the semi-implicit discretization. The numbers for the implicit discretization are nearly the same except for the failures and hence not listed. The average number of PDAS-iterations depend more on the time step than on the mesh. This is an expected behavior since when we use larger time steps the active sets change on a bigger scale than with smaller time steps. In most of the above simulations the maximum number of iterations was needed in the first time step. The reason is that the mean curvature of the interface is high in the beginning of the time evolution, resulting in fast movement of the interface region. Even taking a rather large time step, like for example $\tau=10^{-4}$ for $\pi \varepsilon=0.05$, the maximum number of necessary iterations per time step keeps low and never exceeded 11. The averaged numbers of iterations are much smaller since the time evolution of the interface becomes slow for larger $t$, resulting in only one or two PDAS-iterations.

In Figure 7 we plot the time against the number of used PDAS-iterations per time step as well as against the number of changed vertices per time step for the above simulation with $\pi \varepsilon=0.1, \tau=10^{-5}$, in the semi-implicit and implicit case for an adaptive mesh with $h_{\text {fine }}=0.00552$.

In the first few time steps the evolution smoothens the interfaces and the concave part is moving quickly. These two facts result in an increased number of necessary PDAS-iterations. After that typically two to four iterations are sufficient. The steps where we only need two iterations are optimal in a way that if there is any change in the active set we need at least these two iterations. Only when there are no changes in the sets just one iteration is sufficient. What we can observe in these plots is the expected rise in iteration numbers when there is a big change in the active set. The second peak is due to the disappearance of the bubble in the upper right quadrant. If we use an equidistant mesh for the above example, the results and numbers of 
$u$
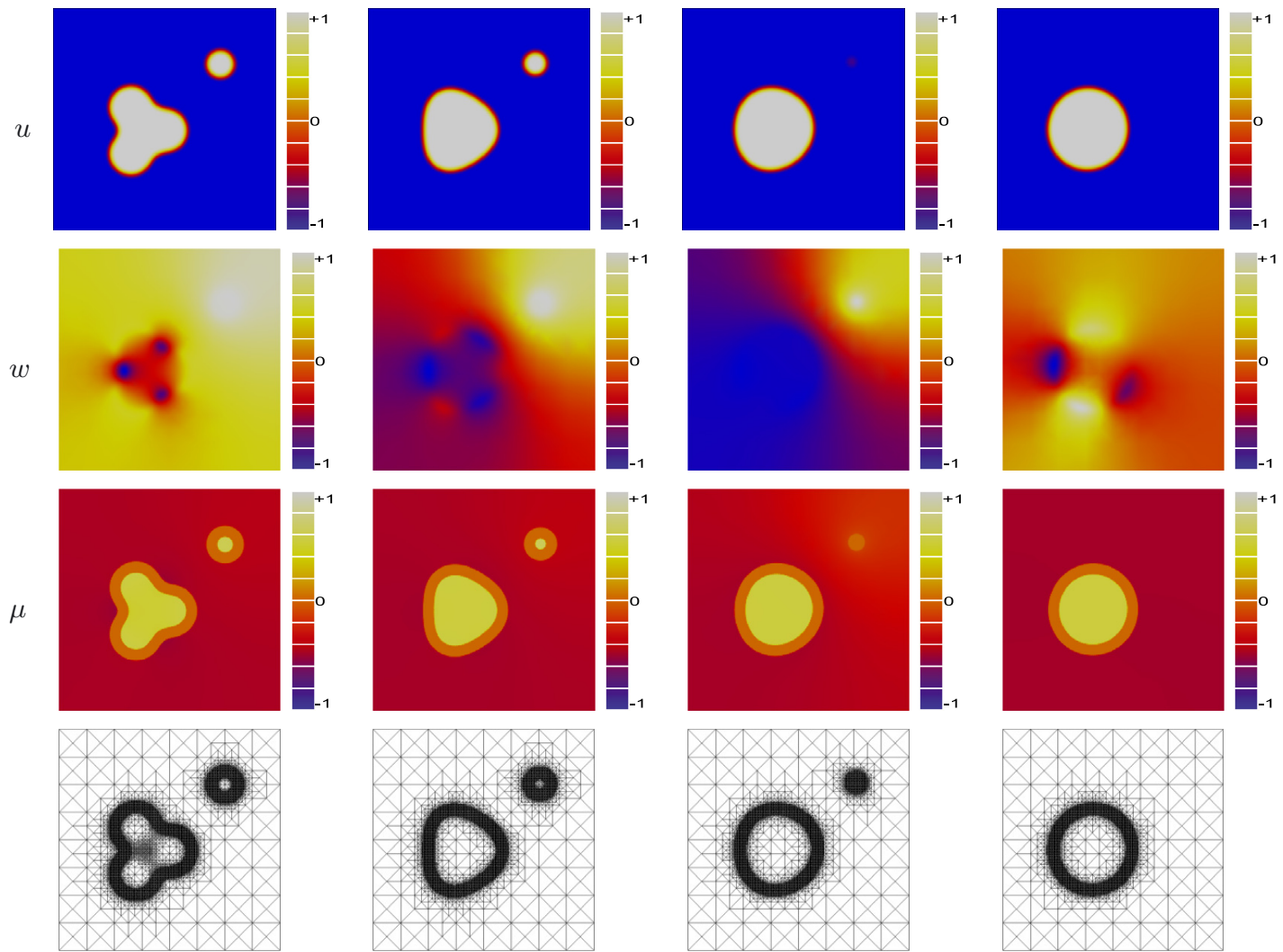

(q) $n=5$

(r) $\mathrm{n}=50$

(s) $\mathrm{n}=100$

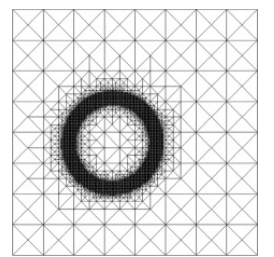

(t) $\mathrm{n}=200$

FiguRE 6. Time evolution of Example 2 with $\pi \varepsilon=0.05$.

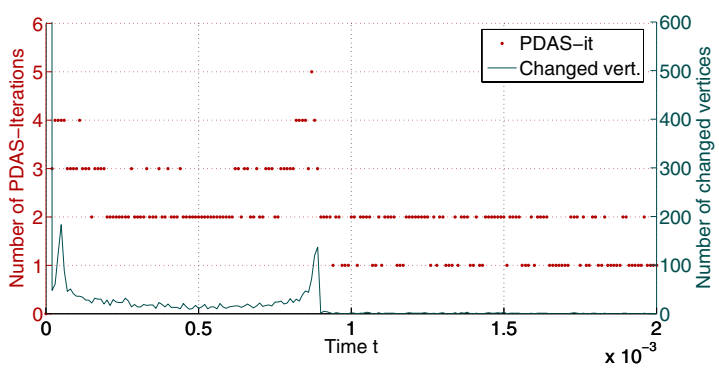

(a) semi-implicit PDAS

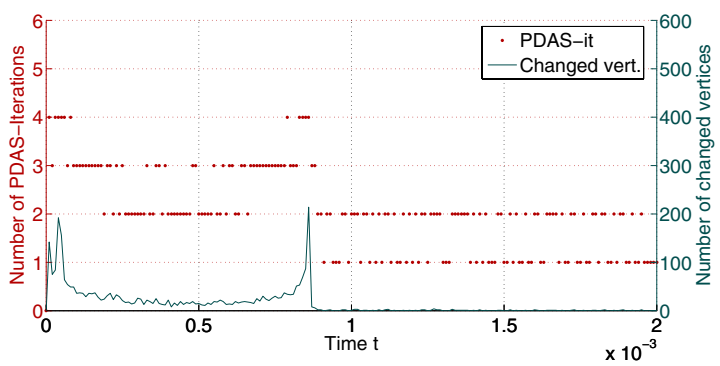

(b) implicit PDAS

FiguRE 7. PDAS-iterations and vertices changing sets per time step.

PDAS-iterations stay nearly the same, although in the adaptive case we have to adapt the starting active set due to a grid change in time (see Initialization of active sets).

However, instead of circa 10 minutes CPU-time for an equidistant grid only $76 \mathrm{~s}$ CPU-time is needed in the adaptive case to determine a solution up to $T=0.02$. Further speed up can be obtained as mentioned before by a different linear algebra solver. 
TABLE 1. CPU-runtimes and iteration counts for Example 2.

\begin{tabular}{|c|c|c|c|c|c|c|c|c|}
\hline \multirow[t]{2}{*}{$\pi \varepsilon$} & \multirow{2}{*}{$\begin{array}{r}h \\
(\mathcal{J})\end{array}$} & \multirow[t]{2}{*}{$\tau$} & \multicolumn{3}{|c|}{ CPU-time in seconds } & \multicolumn{3}{|c|}{ PDAS-iterations } \\
\hline & & & pSOR & $\begin{array}{l}\text { ii-impl. } \\
\text { PDAS }\end{array}$ & $\begin{array}{l}\text { Impl. } \\
\text { PDAS }\end{array}$ & Total & Average & Max. \\
\hline \multirow[t]{9}{*}{0.2} & 0.02210 & $10^{-4}$ & 57.1 & 10.7 & 11.2 & 74 & 3.5 & 5 \\
\hline & $(4225)$ & $10^{-5}$ & 270.9 & 29.7 & 63.6 & 450 & 2.2 & 4 \\
\hline & & $10^{-6}$ & 703.3 & 195.0 & 202.6 & 2958 & 1.5 & 3 \\
\hline & 0.01105 & $10^{-4}$ & 1071.6 & 69.4 & 39.5 & 100 & 4.7 & 7 \\
\hline & $(16641)$ & $10^{-5}$ & 5522.9 & 203.3 & 199.2 & 577 & 2.8 & 4 \\
\hline & & $10^{-6}$ & 13506.2 & 1353.6 & 1325.6 & 3795 & 1.9 & 3 \\
\hline & adaptive & $10^{-4}$ & 5.2 & 2.9 & 2.9 & 72 & 3.4 & 5 \\
\hline & $(\approx 2000)$ & $10^{-5}$ & 23.1 & 17.6 & 17.5 & 447 & 2.2 & 4 \\
\hline & & $10^{-6}$ & 70.2 & 117.9 & 117.8 & 2968 & 1.5 & 3 \\
\hline \multirow[t]{9}{*}{0.1} & 0.01105 & $10^{-4}$ & 1374.6 & 17.9 & 17.8 & 70 & 3.3 & 6 \\
\hline & $(16641)$ & $10^{-5}$ & 4179.5 & 103.4 & 105.6 & 409 & 2.0 & 5 \\
\hline & & $10^{-6}$ & 10111.0 & 793.1 & 727.6 & 2922 & 1.5 & 4 \\
\hline & 0.00552 & $10^{-4}$ & - & 130.3 & 134.5 & 91 & 4.3 & 10 \\
\hline & (66 049) & $10^{-5}$ & - & 750.7 & 754.5 & 524 & 2.6 & 6 \\
\hline & & $10^{-6}$ & 181285.1 & 4905.4 & 4813.2 & 3362 & 1.7 & 5 \\
\hline & adaptive & $10^{-4}$ & 45.1 & 5.1 & 5.1 & 69 & 3.3 & 7 \\
\hline & $(\approx 3600)$ & $10^{-5}$ & 74.5 & 27.7 & 28.2 & 403 & 2.0 & 4 \\
\hline & & $10^{-6}$ & 390.2 & 198.7 & 194.0 & 2897 & 1.4 & 3 \\
\hline \multirow[t]{6}{*}{0.05} & 0.00552 & $10^{-4}$ & 11145.0 & 126.6 & - & 88 & 4.2 & 7 \\
\hline & (66 049) & $10^{-5}$ & 72715.0 & 592.0 & 597.3 & 497 & 2.4 & 6 \\
\hline & & $10^{-6}$ & 192554.4 & 3911.3 & 4013.2 & 3275 & 1.7 & 5 \\
\hline & adaptive & $10^{-4}$ & 737.1 & 13.6 & - & 85 & 4.0 & 7 \\
\hline & $(\approx 7000)$ & $10^{-5}$ & 602.3 & 76.8 & 73.4 & 503 & 2.5 & 6 \\
\hline & & $10^{-6}$ & 1478.2 & 467.6 & 478.1 & 3260 & 1.6 & 5 \\
\hline
\end{tabular}

TABLE 2. CPU-runtimes and iteration counts for Example 2.

\begin{tabular}{rrr|rrr|rrr}
\hline & & & \multicolumn{2}{|c|}{ Splitting algorithm } & \multicolumn{3}{c}{ PDAS-method } \\
$\pi \varepsilon$ & $h$ & $\tau$ & CPU-time & \multicolumn{2}{c}{ Iterations } & \multicolumn{2}{c}{ CPU-time } & Iterations \\
& $(\mathcal{J})$ & & In seconds & Average & Max. & In seconds & Average & Max. \\
\hline 0.2 & 0.02210 & $10^{-4}$ & 105.6 & 428 & 463 & 10.7 & 3.5 & 5 \\
& $(4225)$ & $10^{-5}$ & 1702.0 & 723 & 1500 & 29.7 & 2.2 & 4 \\
& & $10^{-6}$ & 42727.7 & 1839 & 11332 & 195.0 & 1.5 & 3 \\
& adaptive & $10^{-4}$ & 69.3 & 616 & 668 & 2.9 & 3.4 & 5 \\
& $(\approx 2000)$ & $10^{-5}$ & 1224.6 & 1326 & 2258 & 17.6 & 2.2 & 4 \\
& & $10^{-6}$ & \multicolumn{2}{|c|}{ no convergence } & & 117.9 & 1.5 & 3 \\
\hline 0.05 & adaptive & $10^{-4}$ & 2132.5 & 2255 & 2375 & 13.6 & 4.0 & 7 \\
& $(\approx 7000)$ & $10^{-5}$ & 23664.2 & 2792 & max. & 76.8 & 2.5 & 6 \\
\hline
\end{tabular}

Table 2 shows for Example 2 the results of comparative simulations to the splitting method developed in [7] (as mentioned in the above paragraph on the splitting approach solver). The parameter $c$ is fixed and set to 50 and a maximum iteration number of 50000 is used.

\section{Example 3: Random initial data}

In applications one often has to consider initial data which are a random perturbation of a equally distributed concentration $u$. Therefore we give also results on an equally distributed mass on $\Omega=(0,1)^{2}$ with a stochastic distortion. We define $u_{0}(x):=0.5 \cdot \sigma(x)+0.2$, where $\sigma: \Omega \rightarrow[-1,1]$ denotes a random number generator. Consequently there is no pure phase initially, i.e. all vertices are inactive, and the resulting equation 


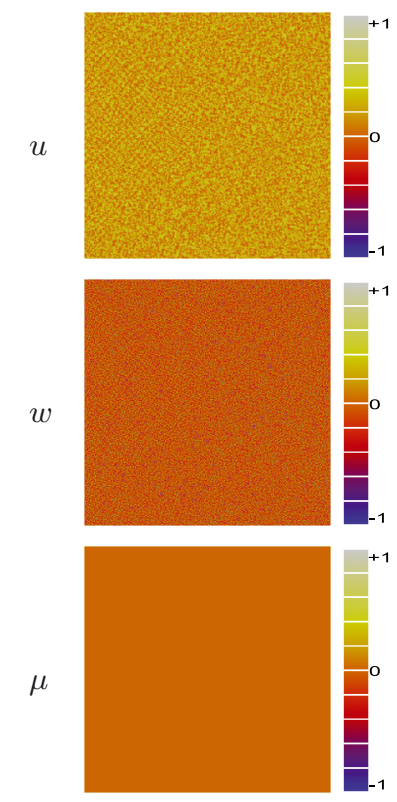

(l) $n=0$
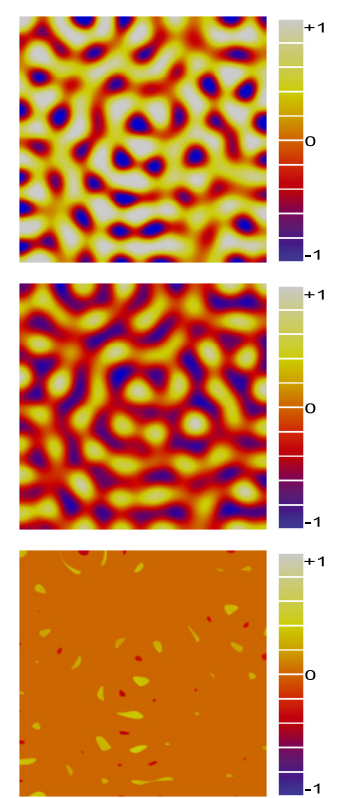

(m) $n=5$
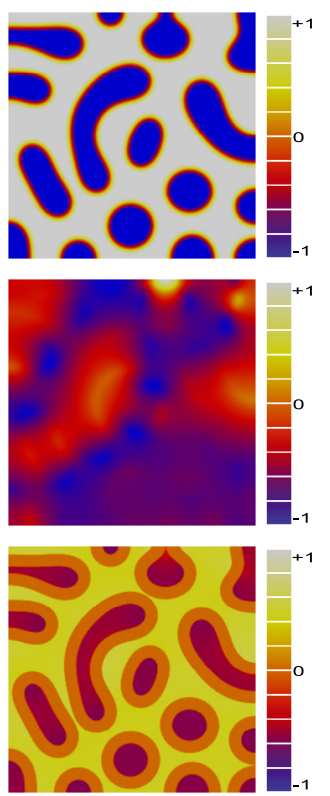

(n) $n=50$
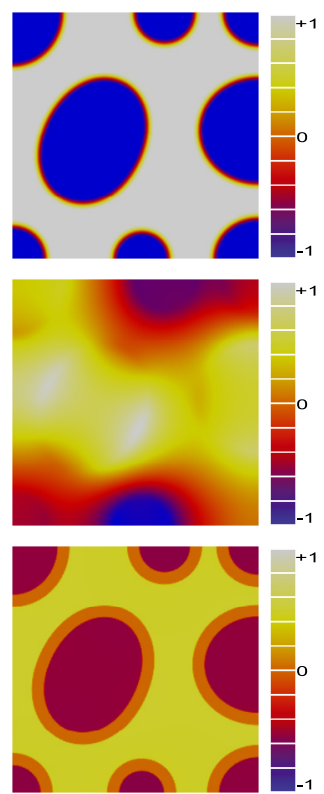

(o) $n=500$

Figure 8. 2D simulation with random initial data.

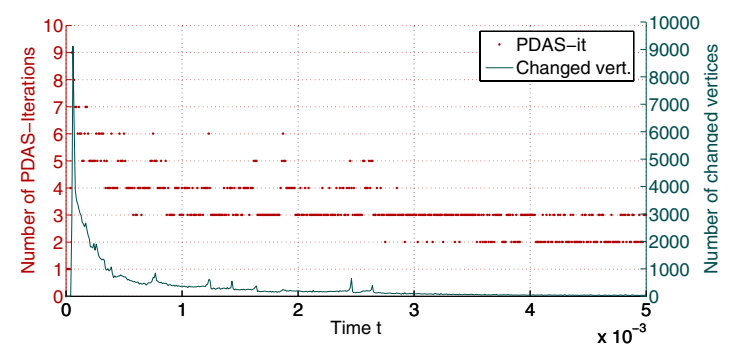

FIGURE 9. PDAS-iterations and vertices changing sets per time step for random initial data.

system (4.8)-(4.10) is as large as possible. For this simulation we used the implicit discretization and a uniform mesh with $h=0.00552, \tau=10^{-5}, T_{\text {end }}=0.005, \pi \varepsilon=0.05$ and $c=10$. In each time step where a new active set emerges, we observe larger values in the Lagrangian multiplier $\mu$, namely $\max |\mu| \approx 20$. Figure 8 shows $u$, $w$ and $\mu$ after $0,5,50$ and 500 time steps. Already after 5 time steps the phase separation can be clearly seen.

In Figure 9 we see that in the early stage of this simulation one PDAS iteration is sufficient since there is no active set present and we just have to solve a linear system. After that a larger number of iterations is necessary because there are quite a few topological changes and a huge amount of vertices is changed from inactive to active. However there have never been more than 10 PDAS-iterations necessary. Afterwards when the interfaces are well developed an average amount of $2-3$ iterations is sufficient. 


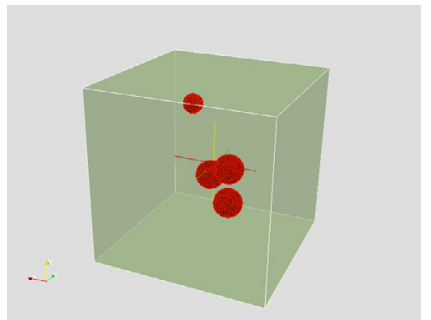

(a) $n=0$

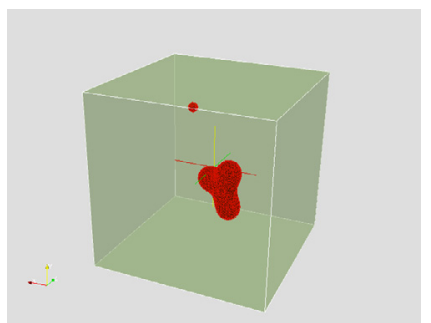

(d) $n=100$

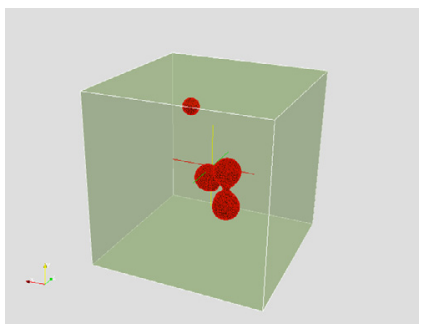

(b) $n=20$

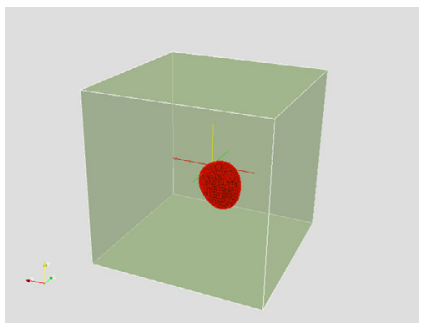

(e) $n=300$

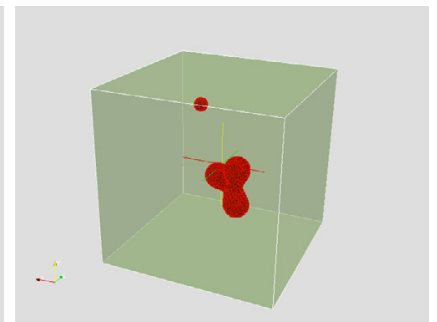

(c) $n=50$

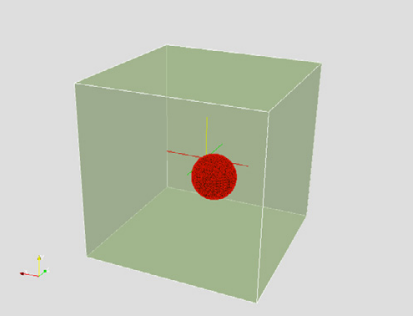

(f) $n=700$

FIGURE 10. 3D simulation with four spheres as initial data.

\section{Example 4: Three dimensional simulation}

Finally we give an example in 3D. Therefore we expand Example 2 to initial data consisting of four balls in $\Omega=(0,1)^{3}$. Figure 10 shows the 0 -level sets of $u$ of such a simulation with $\tau=10^{-5}, \pi \varepsilon=0.1$ and $c=10$ after $0,20,50,100,300$ and 700 time steps on an adaptive mesh with the semi-implicit primal-dual active set solver.

The simulation up to $T_{\mathrm{end}}=0.0007$, i.e. 70 time steps, where a coupled system corresponding to roughly 120000 grid points has to be solved, took $11.8 \mathrm{~h}$ with a total of 184 PDAS-iterations. This is less than half the computation time used by the pSOR method which used $27.6 \mathrm{~h}$. Additional speed up - which is not possible for the pSOR-method- can be obtained by a different linear algebra solver. Even for this three dimensional problem with the topological changes a maximal number of only four PDAS-iterations in each time step is sufficient for the simulation.

\section{REFERENCES}

[1] R.A. Adams, Sobolev spaces, Pure and Applied Mathematics 65. Academic Press, New York-London (1975).

[2] L. Banas and R. Nürnberg, A multigrid method for the Cahn-Hilliard equation with obstacle potential. Appl. Math. Comput. 213 (2009) 290-303.

[3] J.W. Barrett, J.F. Blowey and H. Garcke, Finite element approximation of the Cahn-Hilliard equation with degenerate mobility. SIAM J. Numer. Anal. 37 (1999) 286-318.

[4] J.W. Barrett, R. Nürnberg and V. Styles, Finite element approximation of a void electromigration model. SIAM J. Numer. Anal. 42 (2004) 738-772.

[5] L. Blank, H. Garcke, L. Sarbu and V. Styles, Primal-dual active set methods for Allen-Cahn variational inequalities with non-local constraints. Preprint SPP1253-09-01 (2009).

[6] J.F. Blowey and C.M. Elliott, The Cahn-Hilliard gradient theory for phase separation with nonsmooth free energy. I. Mathematical analysis. Eur. J. Appl. Math. 2 (1991) 233-280.

[7] J.F. Blowey and C.M. Elliott, The Cahn-Hilliard gradient theory for phase separation with nonsmooth free energy. II. Numerical analysis. Eur. J. Appl. Math. 3 (1992) 147-179.

[8] J.F. Blowey and C.M. Elliott, Curvature dependent phase boundary motion and parabolic double obstacle problems, in Degenerate Diffusions, W.-M. Ni, L.A. Peletier and J.L. Vazquez Eds., IMA Vol. Math. Appl. 47, Springer, New York (1993) $19-60$. 
[9] J.F. Blowey and C.M. Elliott, A phase field model with a double obstacle potential, in Motion by mean curvature, G. Buttazzo and A. Visintin Eds., de Gruyter (1994) 1-22.

[10] J.W. Cahn and J.E. Hilliard, Free energy of a nonuniform system. I. Interfacial energy. J. Chem. Phys. 28 (1958) 258-267.

[11] I. Capuzzo Dolcetta, S.F. Vita and R. March, Area-preserving curve-shortening flows: From phase separation to image processing. Interfaces and Free Boundaries 4 (2002) 325-434.

[12] X. Chen, Global asymptotic limit of solutions of the Cahn-Hilliard equation. J. Differential Geom. 44 (1996) $262-311$.

[13] X. Chen, Z. Nashed and L. Qi, Smoothing methods and semismooth methods for nondifferentiable operator equations. SIAM J. Numer. Anal. 38 (2000) 1200-1216.

[14] M. Copetti and C.M. Elliott, Numerical analysis of the Cahn-Hilliard equation with a logarithmic free energy. Numer. Math. 63 (1992) 39-65.

[15] T.A. Davis, Algorithm 832: UMFPACK, an unsymmetric-pattern multifrontal method. ACM Trans. Math. Soft. 30 (2003) 196-199.

[16] T.A. Davis, A column pre-ordering strategy for the unsymmetric-pattern multifrontal method. ACM Trans. Math. Soft. 34 (2003) 165-195.

[17] T.A. Davis and I.S. Duff, An unsymmetric-pattern multifrontal method for sparse LU factorization. SIAM J. Matrix Anal. Appl. 18 (1997) 140-158.

[18] I.S. Duff and J.K. Reid, The multifrontal solution of indefinite sparse symmetric linear. ACM Trans. Math. Soft. 9 (1983) $302-325$.

[19] C.M. Elliott, The Cahn-Hilliard model for the kinetics of phase separation, in Mathematical models for phase change problems, Internat. Ser. Numer. Math. 88, Birkhäuser, Basel (1989).

[20] C.M. Elliott and A.R. Gardiner, One dimensional phase field computations, Numerical Analysis 1993, Proceedings of Dundee Conference, D.F. Griffiths and G.A. Watson Eds., Longman Scientific and Technical (1994) 56-74.

[21] C.M. Elliott and S. Luckhaus, A generalised diffusion equation for phase separation of a multi-component mixture with interfacial free energy. SFB 256, University of Bonn, Preprint 195 (1991).

[22] C.M. Elliott and J. Ockendon, Weak and Variational Methods for Moving Boundary Problems, Pitman Research Notes in Mathematics 59. Pitman (1982).

[23] L.C. Evans, Partial differential equations, Graduate Studies in Mathematics 19. American Mathematical Society, Providence (1998).

[24] A. Friedman, Variational principles and free-boundary problems - Pure and Applied Mathematics. John Wiley \& Sons, Inc., New York (1982).

[25] H. Garcke, Mechanical effects in the Cahn-Hilliard model: A review on mathematical results, in Mathematical Methods and Models in phase transitions, A. Miranvielle Ed., Nova Science Publ. (2005) 43-77.

[26] C. Gräser, Analysis und Approximation der Cahn-Hilliard Gleichung mit Hindernispotential. Diplomarbeit, Freie Universität Berlin, Fachbereich Mathematik und Informatik (2004).

[27] C. Gräser and R. Kornhuber, On preconditioned Uzawa-type iterations for a saddle point problem with inequality constraints, in Domain decomposition methods in science and engineering XVI, Lect. Notes Comput. Sci. Eng. 55, Springer, Berlin (2007) 91-102.

[28] C. Gräser and R. Kornhuber, Nonsmooth Newton methods for set-valued saddle point problems. SIAM J. Numer. Anal. 47 (2009) 1251-1273.

[29] C. Gräser and R. Kornhuber, Multigrid methods for obstacle problems. J. Comput. Math. 27 (2009) 1-44.

[30] M. Hintermüller, K. Ito and K. Kunisch, The primal-dual active set strategy as a semismooth Newton method. SIAM J. Optim. 13 (2002) 865-888.

[31] K. Ito and K. Kunisch, Semi-smooth Newton methods for variational inequalities of the first kind. ESAIM: M2AN 37 (2003) $41-62$.

[32] B.M. Irons, A frontal solution scheme for finite element analysis. Int. J. Numer. Methods Eng. 2 (1970) 5-32.

[33] D. Kinderlehrer and G. Stampacchia, An introduction to variational inequalities and their applications, Pure and Applied Mathematics 88. Academic Press, Inc., New York-London (1980).

[34] E. Kuhl and D.W. Schmid, Computational modeling of mineral unmixing and growth: An application of the Cahn-Hilliard equation. Comp. Mech. 39 (2007) 439-451.

[35] P.-L. Lions and B. Mercier, Splitting algorithms for the sum of two nonlinear operators. SIAM J. Numer. Anal. 16 (1979) 964-979.

[36] J.W.H. Liu, The multifrontal method for sparse matrix solution: Theory and practice. SIAM Rev. 34 (1992) 82-109.

[37] J. Lowengrub and L. Truskinovsky, Quasi-incompressible Cahn-Hilliard fluids and topological transitions. R. Soc. Lond. Proc. Ser. A Math. Phys. Eng. Sci. 454 (1978) 2617-2654.

[38] A. Novick-Cohen, The Cahn-Hilliard equation: mathematical and modeling perspectives. Adv. Math. Sci. Appl. 8 (1998) 965-985.

[39] R.L. Pego, Front migration in the nonlinear Cahn-Hilliard equation. Proc. Roy. Soc. London, Ser. A 422 (1989) 116-133.

[40] A. Schmidt and K.G. Siebert, Design of adaptive finite element software: The finite element toolbox ALBERTA, Lect. Notes Comput. Sci. Eng. 42. Springer, Berlin (2005). 
[41] B. Stoth, Convergence of the Cahn-Hilliard equation to the Mullins-Sekerka problem in spherical symmetry. J. Diff. Equ. 125 (1996) 154-183.

[42] S. Tremaine, On the origin of irregular structure in Saturn's rings. Ast. J. 125 (2003) 894-901.

[43] F. Tröltzsch, Optimale Steuerung partieller Differentialgleichungen: Theorie, Verfahren und Anwendungen. Vieweg Verlag (2005).

[44] S. Zhou and M.Y. Wang, Multimaterial structural topology optimization with a generalized Cahn-Hilliard model of multiphase transition. Struct. Multidisc. Optim. 33 (2007) 89-111. 